\title{
Iron-Catalyzed Homo-Coupling of Simple and Functionalized Arylmagnesium Reagents
}

\author{
G. Cahiez,* C. Chaboche, F. Mahuteau-Betzer, M. Ahr
}

\section{Supporting Information}

\section{General procedures}

Tetrahydrofuran (THF) was Purchased from Merck and freshly distilled from sodium/benzophenone under nitrogen before use.

Iron(III) chloride (anhydrous, 98\%) was Purchased from Acros. It is used as a solution (0.1M) in THF stored under nitrogen atmosphere.

Other reagents were purchased from Acros, Aldrich and Avocado and used as received.

The Grignard reagents $\mathbf{1 a - 1 g}$ and $\mathbf{1 i}-\mathbf{1 j}$ were prepared in THF from the commercially available aryl bromide by the standard method and were titrated according to the procedure published by Watson ${ }^{1}$, excepted in the case of 3-pyridylmagnesium bromide $\mathbf{1 h}$, which was titrated by reaction with isobutyraldehyde. The functionalized aryl Grignard reagents $\mathbf{1 k}-\mathbf{1 q}$ were prepared as described above in the experimental procedures.

${ }^{1} \mathrm{H},{ }^{13} \mathrm{C}$ and ${ }^{19} \mathrm{~F}$ NMR spectra were recorded on JEOL EX-270 and JEOL ECX-400 spectrometers. Chemical shifts $\delta$ are given in ppm from TMS as an internal reference.

Infrared spectra were recorded (liquids were analysed neat between $\mathrm{NaCl}$ plates, solids were analysed in solution in Nujol or as $\mathrm{KBr}$ pellets) on a Fourier transform Nicolet Impact 400 spectrometer at room temperature between 4000 and $600 \mathrm{~cm}^{-1}$. Mass spectra were recorded on a Hewlett-Packard HP 5973 mass spectrometer.

Melting points were measured on a Büchi 530 apparatus and are given without correction.

\section{Homo-coupling of aryl Grignard reagents 1a-1j (Table 1 and 2)}

A dried four-necked flask equipped with a magnetic stirrer and a thermometer was charged under nitrogen with THF $(20 \mathrm{~mL})$, a solution of $\mathrm{FeCl}_{3}$ in THF $(0.1 \mathrm{M}, 3.0 \mathrm{~mL}, 0.3 \mathrm{mmol})$ and 0.6 equivalent of 1,2-dihalogenoethane $(6 \mathrm{mmol})$. A solution of the aryl Grignard reagent 1 in THF (c.a. $1.0 \mathrm{M}, 10 \mathrm{mmol}$ ) was added at once via a syringe to the resulting yellow-green solution. The colour immediately changed to dark brown and the temperature increased. The resulting mixture was stirred at room temperature for $10 \mathrm{~min}$ then hydrolyzed with a $1 \mathrm{M}$ aqueous $\mathrm{HCl}$ solution (30 $\mathrm{mL})$. After extraction with methylene chloride $(3 \times 50 \mathrm{~mL})$, the combined organic layers were dried over anhydrous magnesium sulphate and concentrated under reduced pressure. The residue was purified by flash chromatography on silica gel to give the expected biaryl compound.

\section{Homo-coupling of functionalized aryl Grignard reagents (1k-10) (Table 3)}

A dried four-necked flask equipped with a magnetic stirrer and a thermometer was charged under nitrogen with THF (20 mL) and $10 \mathrm{mmol}$ of iodobenzonitrile (entries 1-3) or ethyl iodobenzoate (entries 4-5). Isopropylmagnesium bromide was added ( $1 \mathrm{M}$ in $\mathrm{THF}, 11 \mathrm{mmol})$ at $-40^{\circ} \mathrm{C}$ to the resulting solution. After stirring at $-40^{\circ} \mathrm{C}$ for $30 \mathrm{~min}$, a solution of $\mathrm{FeCl}_{3}$ in $\mathrm{THF}(0.1 \mathrm{M}, 3.0 \mathrm{~mL}, 0.3$ mmol, ) and 1,2-dihalogenoethane $(10 \mathrm{mmol})$ were added simultaneously and the resulting mixture was stirred at $-40^{\circ} \mathrm{C}$ for $2 \mathrm{~h}$ (entries 1-3) or at room temperature for $1 \mathrm{~h}$ (entries 4-5) then hydrolyzed with a $1 \mathrm{M}$ aqueous $\mathrm{HCl}$ solution $(30 \mathrm{~mL})$. After extraction with methylene chloride $(3 \times 50 \mathrm{~mL})$, the combined organic layers were dried over anhydrous magnesium sulphate and 
concentrated under reduced pressure. The residue was purified by flash chromatography on silica gel to give the expected biaryl compound.

\section{Homo-coupling of 2-nitrophenylmagnesium bromide 1p (Scheme 1)}

A dried four-necked flask equipped with a magnetic stirrer and a thermometer was charged under nitrogen with THF $(30 \mathrm{~mL})$ and 2-iodonitrobenzene $(2.49 \mathrm{~g}, 10 \mathrm{mmol})$. Phenylmagnesium bromide was added $(1.0 \mathrm{M}$ in THF, $10.5 \mathrm{mmol})$ at $-40^{\circ} \mathrm{C}$ to the resulting solution. After stirring at $-40^{\circ} \mathrm{C}$ for 5 min, a solution of $\mathrm{FeCl}_{3}$ in THF $(0.1 \mathrm{M}, 3.0 \mathrm{~mL}, 0.3 \mathrm{mmol})$ and 1,2-dibromoethane $(1.13 \mathrm{~g}, 10$ mmol) were added simultaneously and the resulting mixture was stirred at room temperature for 30 min then hydrolyzed with a $10 \%$ aqueous $\mathrm{NH}_{4} \mathrm{Cl}$ solution $(80 \mathrm{~mL})$. After extraction with ethyl acetate $(3 \times 50 \mathrm{~mL})$, the combined organic layers were dried over anhydrous magnesium sulphate and concentrated under reduced pressure. The residue was purified by chromatography on silica gel (eluent: cyclohexane-AcOEt 70:30) to give $505 \mathrm{mg}$ of $\mathbf{2 p}$ (Yield: 41\%).

\section{Homo-coupling of 2-fluoro-3-carbethoxy-4-pyridylmagnesium bromide 1q (Scheme 2)}

\section{Preparation of 2-fluoro-3-iodopyridine 12}

In a dried four-necked flask equipped with a magnetic stirrer, a thermometer and an addition dropping funnel, LDA was prepared by stirring THF $(250 \mathrm{~mL})$, isopropylamine $(14 \mathrm{~mL}, 0.1 \mathrm{~mol})$ and $n$-butyllithium $(1.60 \mathrm{M}$ in hexane, $62.5 \mathrm{~mL}, 0.1 \mathrm{~mol})$ at $0^{\circ} \mathrm{C}$ for $15 \mathrm{~min}$. The resulting mixture was cooled to $-80^{\circ} \mathrm{C}$ and a solution of 2 -fluoropyridine $(9.71 \mathrm{~g}, 0.10 \mathrm{~mol})$ in THF $(80 \mathrm{~mL})$ was added dropwise at $-75^{\circ} \mathrm{C}$. After stirring at $-75^{\circ} \mathrm{C}$ for $4 \mathrm{~h}$, zinc chloride $(20.4 \mathrm{~g}, 0.15 \mathrm{~mol})$ was added and the temperature was allowed to warm to $0^{\circ} \mathrm{C}$. Iodine was then added and the resulting mixture was stirred at room temperature for $1 \mathrm{~h}$ then hydrolyzed with a $10 \%$ aqueous $\mathrm{NaHSO}_{3}$ solution (100 $\mathrm{mL})$. After extraction with ethyl ether $(3 \times 100 \mathrm{~mL})$, the combined organic layers were washed with a $10 \%$ aqueous solution of $\mathrm{NaHSO}_{3}(2 \times 100 \mathrm{~mL})$ then with brine $(100 \mathrm{~mL})$, dried over anhydrous magnesium sulphate and concentrated under reduced pressure. Crude material was purified by chromatography on silica gel (eluent : cyclohexane-AcOEt 90:10) to give $9 \mathrm{~g}$ of $\mathbf{1 2}$ (Yield: 40\%).

\section{Preparation of 2-fluoro-3-iodo-4-carbethoxypyridine $\mathbf{3 q}$}

In a dried four-necked flask equipped with a magnetic stirrer, a thermometer and an addition dropping funnel, LDA was prepared by stirring THF $(130 \mathrm{~mL})$, isopropylamine $(5.6 \mathrm{~mL}, 40 \mathrm{mmol})$ and $n$-butyllithium $(1.6 \mathrm{M}$ in hexane, $25.0 \mathrm{~mL}, 40 \mathrm{mmol})$ at $0^{\circ} \mathrm{C}$ for $15 \mathrm{~min}$. The resulting mixture was cooled at $-80^{\circ} \mathrm{C}$ and a solution of 2-fluoro-3-iodopyridine (12) $(8.92 \mathrm{~g}, 40 \mathrm{mmol})$ in THF (60 $\mathrm{mL}$ ) was added dropwise at $-75^{\circ} \mathrm{C}$. After stirring at $-75^{\circ} \mathrm{C}$ for $1 \mathrm{~h}$, a solution of magnesium bromide in THF prepared by reacting 1,2-dibromoethane $(9 \mathrm{~g}, 48 \mathrm{mmol})$ with magnesium turnings $(1.22 \mathrm{~g}$, $50 \mathrm{mmol})$ in THF $(120 \mathrm{~mL})$ was added. The resulting solution was stirred at $-75^{\circ} \mathrm{C}$ for 15 min then ethyl cyanoformate $(5,5 \mathrm{~mL}, 56 \mathrm{mmol})$ was added and stirring was continued at room temperature for $1 \mathrm{~h}$. The reaction mixture was then hydrolyzed by brine $(100 \mathrm{~mL})$. After extraction with ethyl acetate $(3 \times 100 \mathrm{~mL})$, the combined organic layers were dried over $\mathrm{MgSO}_{4}$ and concentrated under reduced pressure. Crude material was purified by chromatography on silica gel (eluent: cyclohexane-AcOEt 80:20) to give $7.82 \mathrm{~g}$ of $\mathbf{3 q}$ (Yield : 66\%).

\section{Homo-coupling of 2-fluoro-3-carbethoxy-4-pyridylmagnesium bromide 1q (Scheme 2)}

A dried four-necked flask equipped with a magnetic stirrer and a thermometer was charged under nitrogen with THF (20 mL) and 2-fluoro-3-carbethoxy-4-iodopyridine 3q (2.95 g, $10 \mathrm{mmol})$. The resulting solution was cooled to $-40^{\circ} \mathrm{C}$ then a solution of isopropylmagnesium bromide in THF (c.a. $1 \mathrm{M}, 11 \mathrm{mmol}$ ) was added at $-40^{\circ} \mathrm{C}$. After stirring at $-40^{\circ} \mathrm{C}$ for $30 \mathrm{~min}$, a solution of $\mathrm{FeCl}_{3}$ in $\mathrm{THF}$ $(0.1 \mathrm{M}, 3 \mathrm{~mL}, 0.3 \mathrm{mmol})$ and 1,2-diiodoethane $(1.69 \mathrm{~g}, 6 \mathrm{mmol})$ were added simultaneously and the resulting mixture was stirred at room temperature for $1 \mathrm{~h}$ then hydrolyzed with brine $(50 \mathrm{~mL})$. After 
extraction with chloroform $(3 \times 50 \mathrm{~mL})$, the combined organic layers were washed with a $10 \%$ aqueous $\mathrm{NaHSO}_{3}$ solution $(50 \mathrm{~mL})$, dried over anhydrous magnesium sulphate and concentrated under reduced pressure. The residue was purified by chromatography on silica gel (eluent: cyclohexane-AcOEt 70:30), to give $500 \mathrm{mg}$ of $\mathbf{2 q}$ (Yield: $33 \%$ ).

\section{Intramolecular coupling reaction : preparation of 1,9-dihydrophenanthrene 6 (Scheme 3)}

\section{Preparation of 2-bromo(bromomethyl)benzene $\mathbf{1 3}$}

A flask equipped with a reflux condenser was charged with 2-bromotoluene (51.3 g, $0.3 \mathrm{~mol})$, benzoyl peroxide $(75 \%, 19.4 \mathrm{~g}, 0.06 \mathrm{~mol})$, carbon tetrachloride $(300 \mathrm{~mL})$ and $N$-bromosuccinimide $(53.4 \mathrm{~g}, 0.3 \mathrm{~mol})$. The resulting mixture was refluxed for $2 \mathrm{~h}$ then cooled, washed with a $10 \%$ aqueous $\mathrm{NaHCO}_{3} 10 \%$ solution then with brine $(100 \mathrm{~mL})$, dried over anhydrous magnesium sulphate and concentrated under reduced pressure. Crude material was distilled under reduced pressure (b.p. $74^{\circ} \mathrm{C} / 0.30$ Torr) to give $47 \mathrm{~g}$ of 13 (Yield : $63 \%$ ).

\section{Preparation of 1,2-bis(2-bromophenyl)ethane 14}

A dried four-necked flask equipped with a magnetic stirrer, a thermometer and a reflux condenser was charged under nitrogen with THF $(100 \mathrm{~mL})$ and magnesium turnings $(1.82 \mathrm{~g}, 72 \mathrm{mmol})$. A solution of 2-bromo(bromomethyl)benzene $13(31.6 \mathrm{~g}, 120 \mathrm{mmol})$ in THF (40 mL) was added dropwise under stirring (exothermic) then the resulting suspension was stirred at room temperature for $2 \mathrm{~h}$. The reaction mixture was then hydrolyzed with a $1 \mathrm{M} \mathrm{HCl}$ solution $(150 \mathrm{~mL})$. After extraction with ethyl ether $(3 \times 100 \mathrm{~mL})$, the combined organic layers were dried over anhydrous magnesium sulphate and concentrated under reduced pressure. Crude material was distilled under reduced pressure to eliminate the solvents and the resulting solid was then washed with pentane to give $6.45 \mathrm{~g}$ of 14 (Yield: $32 \%$ ).

\section{Preparation of 1,2-bis(2-iodophenyl)ethane 4}

A dried four-necked flask equipped with a magnetic stirrer, a thermometer and a addition dropping funnel was charged under nitrogen with 1,2-bis(2-bromophenyl)ethane 14 (8.5 g, $25 \mathrm{mmol})$ and THF $(70 \mathrm{~mL})$. The resulting solution was cooled to $-75^{\circ} \mathrm{C}$ and $n$-butyllithium $(1,6 \mathrm{M}$ in hexane, 35.5 $\mathrm{mL}, 55 \mathrm{mmol}$ ) was added at $-75^{\circ} \mathrm{C}$ then the mixture was stirred for $15 \mathrm{~min}$. A solution of iodine $(25.4 \mathrm{~g}, 100 \mathrm{mmol})$ in THF $(110 \mathrm{~mL})$ was added at $-75^{\circ} \mathrm{C}$ and the resulting mixture was stirred at room temperature for $1 \mathrm{~h}$ then hydrolyzed with a $1 \mathrm{M} \mathrm{HCl}$ solution $(150 \mathrm{~mL})$. After extraction with methylene chloride $(3 \times 70 \mathrm{~mL})$, the combined organic layers were washed with a $10 \%$ aqueous $\mathrm{NaHSO}_{3}$ solution $(2 \times 70 \mathrm{~mL})$ then with brine $(70 \mathrm{~mL})$ then dried over anhydrous magnesium sulphate and concentrated under reduced pressure. Crude material was purified by flash chromatography on silica gel (eluent: cyclohexane-methylene chloride 99:1), to give $8.4 \mathrm{~g}$ of 4 (Yield: 77\%).

\section{Intramolecular coupling reaction (Scheme 3)}

A dried four-necked flask equipped with a magnetic stirrer and a thermometer was charged under nitrogen with THF $(15 \mathrm{~mL})$ and 1,2-bis(2-iodophenyl)ethane 4 (1.40 g, $5 \mathrm{mmol})$. A solution of isopropylmagnesium bromide in THF $(1.0 \mathrm{M}, 11 \mathrm{~mL}, 11 \mathrm{mmol}$,) was added then the resulting solution was stirred at room temperature for $60 \mathrm{~min}$. A solution of $\mathrm{FeCl}_{3}$ in $\mathrm{THF}(0.1 \mathrm{M}, 3 \mathrm{~mL}, 0.3$ $\mathrm{mmol})$ and 1,2-dibromoethane $(0.94 \mathrm{~g}, 5 \mathrm{mmol})$ were added simultaneously. The resulting mixture was stirred at room temperature for $60 \mathrm{~min}$ then hydrolyzed with a $1 \mathrm{M} \mathrm{HCl}$ solution $(50 \mathrm{~mL})$. After extraction with methylene chloride $(3 \times 40 \mathrm{~mL})$, the combined organic layers were dried over anhydrous magnesium sulphate and concentrated under reduced pressure. The crude material was purified by chromatography on silica gel (eluent: pentane) to give $0.69 \mathrm{~g}$ of 6 (yield: $76 \%$ ). 


\section{Synthesis of $N$-methylcrinasiadine 11 (Schemes 4 and 5)}

6-bromopiperonal 7, 6-iodopiperonal 8 and 6-iodo-3,4-methylenedioxybenzoic acid 15 were prepared in respectively $54 \%, 80 \%$ and $59 \%$ yields according to procedures described in the litterature. $^{15,16,21}$

\section{Preparation of 2,2'-diiodo-4,5-methylenedioxybenzanilide 16}

A dried four-necked flask equipped with a magnetic stirrer and a thermometer was charged under nitrogen with methylene chloride $(60 \mathrm{~mL}), \mathrm{DMF}(0.1 \mathrm{~mL})$ and 6-iodo-3,4-methylenedioxybenzoic acid 15 (6.0 g, $20 \mathrm{mmol})$. Oxalyl chloride $(3.7 \mathrm{~mL}, 40 \mathrm{mmol})$ was added and, after stirring for $2 \mathrm{~h}$, the resulting solution was concentrated under reduced pressure to give $6.8 \mathrm{~g}$ of the corresponding carboxylic acid chloride as a pale yellow solid.

A dried four-necked flask equipped with a magnetic stirrer, a thermometer and an addition dropping funnel was charged under nitrogen with methylene chloride $(40 \mathrm{~mL}), 2$-iodoaniline $(4.38 \mathrm{~g}, 20$ $\mathrm{mmol})$ and triethylamine $(3.1 \mathrm{~mL}, 22 \mathrm{mmol})$. The mixture was cooled to $0^{\circ} \mathrm{C}$ then a solution of the previously prepared acid chloride in methylene chloride $(50 \mathrm{~mL})$ was added dropwise at $0^{\circ} \mathrm{C}$ and stirring was continued for $3 \mathrm{~h}$ at room temperature. The reaction mixture was then hydrolyzed with water $(100 \mathrm{~mL})$. After extraction with chloroform $(3 \times 80 \mathrm{~mL})$, the combined organic layers were dried over anhydrous magnesium sulphate and concentrated under reduced pressure. Crude material was purified by flash chromatography on silica gel (eluent : $\mathrm{CH}_{2} \mathrm{Cl}_{2}$ ) to give $2.41 \mathrm{~g}$ of $\mathbf{1 6}$ (Yield: $77 \%)$.

\section{Preparation of 2,2'-diiodo-N-methyl-4,5-methylenedioxybenzanilide 9}

A dried four-necked flask equipped with a magnetic stirrer and a thermometer was charged under nitrogen with THF $(30 \mathrm{~mL})$ and sodium hydride $(95 \%, 250 \mathrm{mg}, 9.6 \mathrm{mmol})$. The resulting suspension was cooled to $0^{\circ} \mathrm{C}$ and a solution of 2,2'-diiodo-4,5-methylenedioxybenzanilide 16 $(3.92 \mathrm{~g}, 8 \mathrm{mmol})$ in methylene chloride $(50 \mathrm{~mL})$ was added dropwise at $0^{\circ} \mathrm{C}$. After stirring for 15 min at $0^{\circ} \mathrm{C}$, methyl iodide $(650 \mu \mathrm{L}, 10.4 \mathrm{mmol})$ was added. The resulting mixture was stirred at room temperature for $2 \mathrm{~h}$ then hydrolyzed with water $(60 \mathrm{~mL})$. After extraction with chloroform $(3 \times 60 \mathrm{~mL})$, the combined organic layers were dried over anhydrous magnesium sulphate and concentrated under reduced pressure. Crude material was purified by flash chromatography on silica gel (eluent: $\mathrm{CH}_{2} \mathrm{Cl}_{2}$-AcOEt 97:3) to give $3.47 \mathrm{~g}$ of 9 (Yield: $86 \%$ ).

\section{Preparation of N-methylcrinasiadine 11}

A dried four-necked flask equipped with a magnetic stirrer and a thermometer was charged under nitrogen with THF $(26 \mathrm{~mL})$ and 2,2'-diiodo- $N$-methyl-4,5-methylenedioxybenzanilide 9 (2.03 g, 4.0 $\mathrm{mmol}$ ). The resulting mixture was cooled to $-30^{\circ} \mathrm{C}$ A solution of isopropylmagnesium bromide in THF (c.a. $1.0 \mathrm{M}, 8.8 \mathrm{mmol}$ ) was added at $-25^{\circ} \mathrm{C}$. After stirring at $-25^{\circ} \mathrm{C}$ for $30 \mathrm{~min}$, a solution of $\mathrm{FeCl}_{3}$ in THF $(0.1 \mathrm{M}, 2.4 \mathrm{~mL}, 0.24 \mathrm{mmol}, 6 \mathrm{~mol} \%)$ and 1,2-dibromoethane $(0.75 \mathrm{~g}, 4 \mathrm{mmol})$ were added simultaneously. The resulting mixture was stirred at room temperature for $1 \mathrm{~h}$ then hydrolyzed with brine $(50 \mathrm{~mL})$. After extraction with chloroform $(3 \times 60 \mathrm{~mL})$, the combined organic layers were washed with a $10 \%$ aqueous $\mathrm{NaHSO}_{3}$ solution $(50 \mathrm{~mL})$, dried over anhydrous magnesium sulphate and concentrated under reduced pressure. Crude material was purified by flash chromatography on silica gel (eluent: cyclohexane-AcOEt 60:40) then recristallized from ethanol to give $400 \mathrm{mg}$ of $N$-methylcrinasiadine (Yield: 39\%). 


\section{SPECTROSCOPIC DATA}

All the known compounds gave satisfactory spectroscopic values which are given below and are analogue to spectroscopic data reported in the litterature. Elemental analysis or HRMS are given for each new compound.

Biphenyl ${ }^{2}$ (2a): white solid; m.p. $67-69^{\circ} \mathrm{C}$ (Lit. $\left.69-71^{\circ} \mathrm{C}\right)$; IR $(\mathbf{K B r}) v=3031,1941,1880,1573$, 1481, 1342, 1173, 1081, 902, 743, $702 \mathrm{~cm}^{-1}$; ${ }^{\mathbf{H}} \mathbf{H} \mathbf{~ N M R}\left(\mathbf{4 0 0} \mathbf{~ M H z}, \mathbf{C D C l}_{3}, \mathbf{2 5}^{\circ} \mathbf{C}\right) \delta: 7.34(1 \mathrm{H}, \mathrm{t}$, $\left.\mathrm{J}^{3}(\mathrm{H})=7.3 \mathrm{~Hz}\right), 7.43\left(2 \mathrm{H}, \mathrm{d}, \mathrm{J}^{3}(\mathrm{H})=7.8 \mathrm{~Hz}\right), 7.59\left(2 \mathrm{H}, \mathrm{d}, \mathrm{J}^{3}(\mathrm{H})=7.4 \mathrm{~Hz}\right) ;{ }^{13} \mathbf{C}$ NMR (100 MHz, CDCl $\mathbf{~}_{3}$, $\left.\mathbf{2 5}^{\circ} \mathbf{C}\right) \delta: 127.1(\mathrm{CH}), 127.2(\mathrm{CH}), 128.7(\mathrm{CH}), 141.2(\mathrm{C})$; MS (EI, 70eV) $\mathbf{m} / \mathbf{z}(\%): 154(100)$ $\left[\mathrm{M}^{+}\right], 76(15)\left[\mathrm{M}^{+}-\mathrm{C}_{6} \mathrm{H}_{6}\right]$.

4,4'-Dimethoxybiphenyl $\mathbf{l}^{\mathbf{2}}$ (2b): white solid; m.p. $178-179^{\circ} \mathrm{C}$ (Lit. $\left.179-180^{\circ} \mathrm{C}\right)$; IR (KBr) $v=1614$, 1506, 1276, 1250, 1178, 1040, 1015, 825, $805 \mathrm{~cm}^{-1} ;{ }^{\mathbf{1}} \mathbf{H}$ NMR (400 $\left.\mathbf{~ M H z , ~} \mathbf{C D C l}_{\mathbf{3}}, \mathbf{2 5}^{\circ} \mathbf{C}\right) \delta: 3.83$ $(3 \mathrm{H}, \mathrm{s}), 6.95\left(2 \mathrm{H}, \mathrm{dm}, \mathrm{J}^{3}(\mathrm{H})=9.1 \mathrm{~Hz}\right), 7.47\left(2 \mathrm{H}, \mathrm{dm}, \mathrm{J}^{3}(\mathrm{H})=9.2 \mathrm{~Hz}\right) ;{ }^{13} \mathbf{C}$ NMR (100 MHz, CDCl , $\left.\mathbf{2 5}^{\circ} \mathbf{C}\right) \delta: 55.3\left(\mathrm{CH}_{3}\right), 114.1(\mathrm{CH}), 127.7(\mathrm{CH}), 133.4(\mathrm{C}), 158.7(\mathrm{C}) ; \mathbf{M S}(\mathbf{E I}, \mathbf{7 0 e V}) \mathbf{m} / \mathbf{z}(\mathbf{\%}): 214$ (100) $\left[\mathrm{M}^{+}\right], 199(85)\left[\mathrm{M}^{+}-\mathrm{CH}_{3}\right], 171(26), 156(09), 139(08), 128(15)$.

3,3'-Dimethoxybiphenyl ${ }^{3}$ (2c): colourless oil; IR (Neat) $v=3058,3006,2950,2838,1598,1575$, 1481, 1415, 1279, 1237, 1204, 1171, 1052, 1035, 857, 777, $702 \mathrm{~cm}^{-1} ;{ }^{1} \mathbf{H}$ NMR (400 MHz, CDCl $\mathbf{H}_{3}$, $\left.\mathbf{2 5}^{\circ} \mathbf{C}\right) \delta: 3.82(3 \mathrm{H}, \mathrm{s}), 6.88\left(1 \mathrm{H}, \mathrm{dd}, \mathrm{J}^{3}=8.2 \mathrm{~Hz}, \mathrm{~J}^{4}=2.3 \mathrm{~Hz}\right), 7.12\left(1 \mathrm{H}, \mathrm{dd}, \mathrm{J}^{4}=2.3 \mathrm{~Hz}, \mathrm{~J}^{4}=1.8 \mathrm{~Hz}\right), 7.16$ $\left(1 \mathrm{H}, \mathrm{d}, \mathrm{J}^{3}=7.8 \mathrm{~Hz}\right), 7.32\left(1 \mathrm{H}, \mathrm{dd}, \mathrm{J}^{3}=8.2 \mathrm{~Hz}, \mathrm{~J}^{3}=7.8 \mathrm{~Hz}\right) ;{ }^{13} \mathbf{C} \mathbf{N M R}\left(\mathbf{1 0 0} \mathbf{M H z}, \mathbf{C D C l}_{\mathbf{3}}, \mathbf{2 5}^{\circ} \mathbf{C}\right) \delta$ : 55.2, 112.7, 112.9, 119.6, 129.7, 142.5, 159.8; MS (EI, 70eV) $\mathbf{~ m / z ~ ( \% ) ~ : ~} 214(100)\left[\mathrm{M}^{+\bullet}\right], 171(23)$, 153 (07), 139 (10), 128 (15), 115 (06); Elemental Analysis calculated: C 78.48 \%, H 6.59 \%, O $14.93 \%$; found : C $78.44 \%$, H $6.35 \%$.

4,4'-bis(dimethylamino)biphenyl ${ }^{4}$ (2d): white solid; m.p. $193-194^{\circ} \mathrm{C}$ (Lit. $\left.195^{\circ} \mathrm{C}\right)$; IR (Nujol) $v=$ 2720, 1609, 1352, 1224, 1189, 1055, 943, 805, $728 \mathrm{~cm}^{-1}$; ${ }^{\mathbf{1}} \mathbf{H}$ NMR (400 $\left.\mathbf{~ M H z , ~} \mathbf{C D C l}_{\mathbf{3}}, \mathbf{2 5}^{\circ} \mathbf{C}\right) \delta$ : $2.96(6 \mathrm{H}, \mathrm{s}), 6.79\left(2 \mathrm{H}, \mathrm{d}, \mathrm{J}^{3}(\mathrm{H})=8.2 \mathrm{~Hz}\right), 7.45\left(2 \mathrm{H}, \mathrm{d}, \mathrm{J}^{3}(\mathrm{H})=9.2 \mathrm{~Hz}\right) ;{ }^{13} \mathbf{C}$ NMR (100 MHz, CDCl $\mathbf{~}_{3}$, $\left.\mathbf{2 5}^{\circ} \mathbf{C}\right) \delta: 40.8\left(\mathrm{CH}_{3}\right), 113.1(\mathrm{CH}), 126.9(\mathrm{CH}), 129.8(\mathrm{C}), 149.2(\mathrm{C}) ; \mathbf{M S}(\mathbf{E I}, \mathbf{7 0 e V}) \mathbf{~ m} / \mathbf{z}(\%): 240$ (100) $\left[\mathrm{M}^{+}\right], 225(30)\left[\mathrm{M}^{+}-\mathrm{CH}_{3}\right], 152(10), 119(24)$.

4,4'-difluorobiphenyl $\mathbf{2}^{\mathbf{2}}$ (2e): white solid; m.p. $89-90^{\circ} \mathrm{C}\left(\right.$ Litt. $\left.90-93^{\circ} \mathrm{C}\right)$; IR (Nujol) $v=3073,1890$, 1603, 1501, 1322, 1235, 1112, $825 \mathrm{~cm}^{-1} ;{ }^{1} \mathbf{H}$ NMR (400 MHz, $\left.\mathbf{C D C l}_{3}, \mathbf{2 5}^{\circ} \mathbf{C}\right) \delta: 7.07-7.15(2 \mathrm{H}, \mathrm{m})$, 7.44-7,51 (2H, m); ${ }^{13} \mathbf{C}$ NMR (100 MHz, $\left.\mathbf{C D C l}_{3}, \mathbf{2 5}^{\circ} \mathbf{C}\right) \delta: 115.7\left(\mathrm{~d}, \mathrm{~J}^{2}(\mathrm{C}-\mathrm{F})=21,1 \mathrm{~Hz}\right), 128.6(\mathrm{~d}$, $\left.\mathrm{J}^{3}(\mathrm{C}-\mathrm{F})=8,6 \mathrm{~Hz}\right), 136.4\left(\mathrm{~d}, \mathrm{~J}^{4}(\mathrm{C}-\mathrm{F})=2,9 \mathrm{~Hz}\right), 162.4\left(\mathrm{~d}, \mathrm{~J}^{1}(\mathrm{C}-\mathrm{F})=246,6 \mathrm{~Hz}\right) ;{ }^{19} \mathbf{F}$ NMR (376 MHz, $\left.\mathbf{C D C l}_{3}, \mathbf{2 5}^{\circ} \mathbf{C}\right) \delta:-115,6(\mathrm{~s}) ; \mathbf{M S}(\mathbf{I E}, \mathbf{7 0 e V}) \mathbf{~ m} / \mathbf{z}(\%): 190(100)\left[\mathrm{M}^{+\bullet}\right], 170(09)\left[\mathrm{M}^{+\bullet}-\mathrm{HF}\right]$.

1,1'-binaphthyl $\mathbf{2}^{\mathbf{( 2 f}}$ ): white solid; m.p. $143-144^{\circ} \mathrm{C}\left(\right.$ Lit. $\left.158-160^{\circ} \mathrm{C}\right)$; IR (Nujol) $\boldsymbol{v}=3048,1583$, 1506, 1015, 968, 805, 779, $682 \mathrm{~cm}^{-1}$; ${ }^{1} \mathbf{H}$ NMR (400 $\left.\mathbf{M H z} \mathbf{C D C l}_{3}, \mathbf{2 5}^{\circ} \mathbf{C}\right) \delta: 7.24-7.31(1 \mathrm{H}, \mathrm{m})$, $7.39\left(1 \mathrm{H}, \mathrm{d}, \mathrm{J}^{3}(\mathrm{H})=8.2 \mathrm{~Hz}\right), 7.42-7.52(2 \mathrm{H}, \mathrm{m}), 7.58\left(1 \mathrm{H}, \mathrm{t}, \mathrm{J}^{3}(\mathrm{H})=7.6 \mathrm{~Hz}\right), 7.91-7.98(2 \mathrm{H}, \mathrm{m}) ;{ }^{13} \mathbf{C}$ NMR (100 MHz, $\left.\mathbf{C D C l}_{3}, \mathbf{2 5}^{\circ} \mathbf{C}\right) \delta: 125.5(\mathrm{CH}), 125.9(\mathrm{CH}), 126.1(\mathrm{CH}), 126.7(\mathrm{CH}), 127.9(\mathrm{CH})$, $128.0(\mathrm{CH}), 128.3(\mathrm{CH}), 133.0(\mathrm{C}), 133.6(\mathrm{C}), 138.6(\mathrm{C})$; MS (EI, 70eV) m/z (\%) : $254(100)\left[\mathrm{M}^{+}\right]$, $126(16)$.

2,2'-binaphtyl $\mathbf{2}^{\mathbf{2}} \mathbf{2 g}$ ): white solid; m.p. $183-184^{\circ} \mathrm{C}$ (Lit. $183-185^{\circ} \mathrm{C}$ ); IR (Nujol) $v=3048,1594$, 1276, 1132, 968, 886, 856, 810, $738 \mathrm{~cm}^{-1} ;{ }^{1} \mathbf{H}$ NMR (400 $\left.\mathbf{M H z}, \mathbf{C D C l}_{3}, \mathbf{2 5}^{\circ} \mathbf{C}\right) \delta: 7.46-7.56(2 \mathrm{H}$, m), 7.86-7.98 (4H, m), $\left.8.17(1 \mathrm{H}, \mathrm{s}) ;{ }^{\mathbf{1 3}} \mathbf{C} \mathbf{~ N M R ~ ( 1 0 0 ~} \mathbf{~ M H z}, \mathbf{C D C l}_{\mathbf{3}}, \mathbf{2 5}^{\circ} \mathbf{C}\right) \delta: 125.7(\mathrm{CH}), 126.0$ $(\mathrm{CH}), 126.1(\mathrm{CH}), 126.3(\mathrm{CH}), 127.7(\mathrm{CH}), 128.2(\mathrm{CH}), 128.5(\mathrm{CH}), 132.7(\mathrm{C}), 133.7(\mathrm{C}), 138.4$ (C); MS (EI, 70eV) m/z (\%) : 254 (100) $\left[\mathrm{M}^{+}\right], 126$ (16). 
3,3'-bipyridyl ${ }^{\mathbf{5}}$ (2h): orange oil; m.p. $230-231^{\circ} \mathrm{C}$ (Picrate) $\left(\right.$ Lit. $\left.232^{\circ} \mathrm{C}\right)$; IR (Neat) $v=3032,1588$, 1562, 1465, 1424, 1399, 1332, 1199, 1025, 999, 794, $717 \mathrm{~cm}^{-1} ;{ }^{1} \mathbf{H}$ NMR (270 $\left.\mathbf{~ M H z}, \mathbf{C D C l}_{3}, \mathbf{2 5}^{\circ} \mathbf{C}\right)$ $\delta: 7.38-7.45(1 \mathrm{H}, \mathrm{m}), 7.89\left(1 \mathrm{H}, \mathrm{ddd}, \mathrm{J}^{3}=7.8 \mathrm{~Hz}, \mathrm{~J}^{4}=2.3 \mathrm{~Hz}, \mathrm{~J}^{4}=1.8 \mathrm{~Hz}\right), 8.65\left(1 \mathrm{H}, \mathrm{dd}, \mathrm{J}^{3}=4.8 \mathrm{~Hz}\right.$, $\left.\mathrm{J}^{4}=1.8 \mathrm{~Hz}\right), 8.85\left(2 \mathrm{H}, \mathrm{d}, \mathrm{J}^{4}=2.3 \mathrm{~Hz}\right) ;{ }^{13} \mathbf{C}$ NMR (68 $\left.\mathbf{M H z}, \mathbf{C D C l}_{3}, \mathbf{2 5}^{\circ} \mathbf{C}\right) \delta: 123.5,133.1,134.1$,

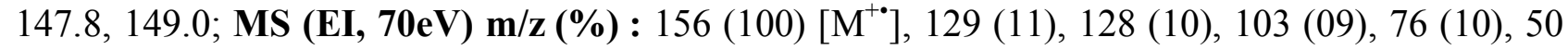
(08).

2,2'-Dimethoxybiphenyl $\mathbf{l}^{6}(\mathbf{2 i})$ : white solid; m.p. $155-156^{\circ} \mathrm{C}$ (Lit. 154-156 ${ }^{\circ} \mathrm{C}$ ); IR (Nujol) $v=$ 1588, 1506, 1429, 1310, 1281, 1255, 1240, 1220, 1168, 1112, 1061, 1025, 999, 933, $764 \mathrm{~cm}^{-1} ;{ }^{1} \mathbf{H}$ NMR (400 MHz, $\left.\mathbf{C D C l}_{3}, \mathbf{2 5}^{\circ} \mathrm{C}\right) \delta: 3.77(3 \mathrm{H}, \mathrm{s}), 6.93-7.04(2 \mathrm{H}, \mathrm{m}), 7.21-7.38(2 \mathrm{H}, \mathrm{m}) ;{ }^{13} \mathbf{C}$ NMR $\left(100 \mathrm{MHz}, \mathbf{C D C l}_{3}, \mathbf{2 5}^{\circ} \mathrm{C}\right) \delta: 55.6\left(\mathrm{CH}_{3}\right), 111.0(\mathrm{CH}), 120.3(\mathrm{CH}), 127.7(\mathrm{C}), 128.6(\mathrm{CH}), 131.4$ (CH), 157.0 (C); MS (EI, 70eV) m/z (\%) : 214 (100) $\left[\mathrm{M}^{+}\right], 199$ (15) $\left[\mathrm{M}^{+}-\mathrm{CH}_{3}\right], 184$ (32), 168 (21), 155 (07), 139 (12), 115 (07), 102 (04), 91 (03).

2,2',4,4',6,6'-hexamethylbiphenyl ${ }^{7}$ (2j): white solid; m.p. 99-100 ${ }^{\circ} \mathrm{C}$ (Lit. $\left.100^{\circ} \mathrm{C}\right)$; IR (Nujol) $\boldsymbol{v}=$ 2730, 1609, 1009, $851 \mathrm{~cm}^{-1}{ }^{\mathbf{1}} \mathbf{H}$ NMR (400 MHz, $\left.\mathbf{C D C l}_{\mathbf{3}}, \mathbf{2 5}^{\circ} \mathbf{C}\right) \delta: 1.86(6 \mathrm{H}, \mathrm{s}), 2.33(3 \mathrm{H}, \mathrm{s}), 6.93$ $(2 \mathrm{H}, \mathrm{s}) ;{ }^{13} \mathbf{C}$ NMR (100 MHz, $\left.\mathbf{C D C l}_{3}, \mathbf{2 5}^{\circ} \mathbf{C}\right) \delta: 19.8\left(\mathrm{CH}_{3}\right), 21.1\left(\mathrm{CH}_{3}\right), 128.2(\mathrm{CH}), 135.5(\mathrm{C})$, 136.0 (C), 137.0 (C) ; MS (EI, 70eV) $\boldsymbol{m} / \boldsymbol{z}(\%): 238(86)\left[\mathrm{M}^{+}\right], 223(100)\left[\mathrm{M}^{+}-\mathrm{CH}_{3}\right], 208(47), 193$ (34), $178(11), 165$ (09).

4,4'-dicyanobiphenyl $\mathbf{8}^{\mathbf{8}}(\mathbf{2 k})$ : white solid ; m.p. $233-234^{\circ} \mathrm{C}$ (Lit. $\left.233-234^{\circ} \mathrm{C}\right)$; IR (KBr) $v=2227$, 1604, 1490, 1397, 1180, 859, 818, $545 \mathrm{~cm}^{-1} ;{ }^{1} \mathbf{H}$ NMR (400 MHz, $\left.\mathbf{C D C l}_{3}, \mathbf{2 5}^{\circ} \mathbf{C}\right) \delta: 7.68-7.75(2 \mathrm{H}$, $\mathrm{m}), 7.75-7.82(2 \mathrm{H}) ;{ }^{13} \mathbf{C}$ NMR (100 $\left.\mathbf{~ M H z}, \mathbf{C D C l}_{3}, \mathbf{2 5}^{\circ} \mathbf{C}\right) \delta: 112.4,118.4,127.9,132.9,143.5 ; \mathbf{M S}$ (EI, 70eV) $\mathbf{m} / \mathbf{z}(\%): 204(100)\left[\mathrm{M}^{+\bullet}\right], 177(10)\left[\mathrm{M}^{+\bullet}-\mathrm{HCN}\right]$.

3,3'-dicyanobiphenyl'(2l): white solid ; m.p. $200-201^{\circ} \mathrm{C}$; IR $(\mathbf{K B r}) \boldsymbol{v}=2232,1602,1580,1475$, 1401, 1336, 1270, 1195, 1101, 898, 813, 791, 688, 589, $565 \mathrm{~cm}^{-1} ;{ }^{1} \mathbf{H}_{\text {NMR }}\left(400 \mathrm{MHz}, \mathbf{C D C l}_{3}\right.$, $\left.\mathbf{2 5}^{\circ} \mathbf{C}\right) \delta: 7.62\left(1 \mathrm{H}, \mathrm{dd}, \mathrm{J}^{3}=7.8 \mathrm{~Hz}, \mathrm{~J}^{3}=7.8 \mathrm{~Hz}\right), 7.72\left(1 \mathrm{H}, \mathrm{ddd}, \mathrm{J}^{3}=7.8 \mathrm{~Hz}, \mathrm{~J}^{4}=1.4 \mathrm{~Hz}, \mathrm{~J}^{4}=1.4 \mathrm{~Hz}\right), 7.81$ $\left(1 \mathrm{H}, \mathrm{ddd}, \mathrm{J}^{3}=7.8 \mathrm{~Hz}, \mathrm{~J}^{4}=1.4 \mathrm{~Hz}, \mathrm{~J}^{4}=1.4 \mathrm{~Hz}\right), 7.85\left(1 \mathrm{H}, \mathrm{d}, \mathrm{J}^{4}=1.4 \mathrm{~Hz}\right) ;{ }^{13} \mathbf{C}$ NMR (100 MHz, CDCl $\mathbf{~}_{3}$, $\left.\mathbf{2 5}^{\circ} \mathbf{C}\right) \delta: 113.4,118.3,130.0,130.6,131.4,131.8,140.1 ;$ MS (EI, 70eV) $\mathbf{~ m} / \mathbf{z}(\%): 204(100)$ $\left[\mathrm{M}^{+\bullet}\right], 177(12)\left[\mathrm{M}^{+\bullet}-\mathrm{HCN}\right], 150(04)\left[\mathrm{M}^{+\bullet}-2 \mathrm{HCN}\right]$.

2,2'-dicyanobiphenyl ${ }^{\mathbf{1 0}}$ (2m): white solid ; m.p. $173-174^{\circ} \mathrm{C}$; IR $(\mathbf{K B r}) \boldsymbol{v}=2227,1594,1562$, 1482, 1429, 1266, 1200, 1064, 975, 768, 735, 562, $540 \mathrm{~cm}^{-1}$; ${ }^{1} \mathbf{H}$ NMR (400 MHz, $\left.\mathbf{C D C l}_{3}, \mathbf{2 5}^{\circ} \mathbf{C}\right)$ $\delta: 7.55-7.61(2 \mathrm{H}, \mathrm{m}), 7.73\left(1 \mathrm{H}, \mathrm{ddd}, \mathrm{J}^{3}=7.8 \mathrm{~Hz}, \mathrm{~J}^{3}=7.8 \mathrm{~Hz}, \mathrm{~J}^{4}=1.4 \mathrm{~Hz}\right), 7.81-7.86(1 \mathrm{H}, \mathrm{m}) ;{ }^{13} \mathbf{C} \mathbf{N M R}$ (100 MHz, $\left.\mathbf{C D C l}_{3}, \mathbf{2 5}^{\circ} \mathbf{C}\right) \delta: 112.3,117.5,129.2,130.5,132.8,133.5,141.5 ;$ MS (EI, 70eV) $\mathbf{m} / \mathbf{z}(\%): 204(100)\left[\mathrm{M}^{+\bullet}\right], 177(18)\left[\mathrm{M}^{+\bullet}-\mathrm{HCN}\right], 150(06)\left[\mathrm{M}^{+\bullet}-2 \mathrm{HCN}\right]$; Elemental Analysis calculated : C $82.33 \%$, H $3.95 \%$, N $13.72 \%$; found C $82.16 \%$, H $3.78 \%$, N $13.63 \%$.

4,4'-dicarbethoxybiphenyl ${ }^{\mathbf{8}}(\mathbf{2 n})$ : white solid ; m.p. $223-224^{\circ} \mathrm{C}$ (Lit. 224-226 ${ }^{\circ} \mathrm{C}$ ) ; IR (KBr) v $=1715,1608,1561,1485,1453,1401,1373,1279,1185,1110,1026,1007,885,852,758,697$ $\mathrm{cm}^{-1}{ }^{1} \mathbf{H}$ NMR (400 MHz, $\left.\mathbf{C D C l}_{3}, \mathbf{2 5}^{\circ} \mathbf{C}\right) \delta: 1.42\left(3 \mathrm{H}, \mathrm{t}, \mathrm{J}^{3}=7.3 \mathrm{~Hz}\right), 4.41\left(2 \mathrm{H}, \mathrm{q}, \mathrm{J}^{3}=7.3 \mathrm{~Hz}\right), 7.68$ $\left(2 \mathrm{H}, \mathrm{d}, \mathrm{J}^{3}=8.7 \mathrm{~Hz}\right), 8.14\left(2 \mathrm{H}, \mathrm{d}, \mathrm{J}^{3}=8.7 \mathrm{~Hz}\right) ;{ }^{13} \mathbf{C}$ NMR (100 $\left.\mathbf{M H z}, \mathbf{C D C l}_{\mathbf{3}}, \mathbf{2 5}^{\circ} \mathbf{C}\right) \delta: 14.3,61.0$, 127.1, 129.9, 130.1, 144.2, 166.2 ; MS (EI, 70eV) $298(61)\left[\mathrm{M}^{+\bullet}\right], 270(07)\left[\mathrm{M}^{+\bullet}-\mathrm{CO}\right], 253(100)$ $\left[\mathrm{M}^{+\bullet}-\mathrm{OC}_{2} \mathrm{H}_{5}\right], 242(11), 225(29)\left[\mathrm{M}^{+\bullet}-\mathrm{COOC}_{2} \mathrm{H}_{5}\right], 180,(05), 152(38)\left[\mathrm{M}^{+\bullet}-2 \mathrm{COOC}_{2} \mathrm{H}_{5}\right], 104(05)$, 76 (05).

3,3'-dicarbethoxybiphenyl ${ }^{\mathbf{1 1}}$ (2o): white solid ; m.p. $68-69^{\circ} \mathrm{C}$; IR (KBr) $v=3002,2978,1730$, 1594, 1479, 1391, 1372, 1309, 1246, 1106, 1087, 1029, 893, 743, $695 \mathrm{~cm}^{-1}$; ${ }^{1}$ H NMR (400 MHz, $\left.\mathbf{C D C l}_{3}, \mathbf{2 5}^{\circ} \mathbf{C}\right) \delta: 1.43\left(3 \mathrm{H}, \mathrm{t}, \mathrm{J}^{3}=7.3 \mathrm{~Hz}\right), 4.42\left(2 \mathrm{H}, \mathrm{q}, \mathrm{J}^{3}=7.3 \mathrm{~Hz}\right), 7.54\left(1 \mathrm{H}, \mathrm{dd}, \mathrm{J}^{3}=7.8 \mathrm{~Hz}\right.$, $\left.\mathrm{J}^{3}=7.8 \mathrm{~Hz}\right), 7.81\left(1 \mathrm{H}, \mathrm{ddd}, \mathrm{J}^{3}=7.8 \mathrm{~Hz}, \mathrm{~J}^{4}=1.8 \mathrm{~Hz}, \mathrm{~J}^{4}=1.8 \mathrm{~Hz}\right), 8.06\left(1 \mathrm{H}, \mathrm{ddd}, \mathrm{J}^{3}=7.8 \mathrm{~Hz}, \mathrm{~J}^{4}=1.8 \mathrm{~Hz}\right.$, $\left.\mathrm{J}^{4}=1.8 \mathrm{~Hz}\right), 8.31\left(1 \mathrm{H}, \mathrm{dd}, \mathrm{J}^{4}=1.8 \mathrm{~Hz}, \mathrm{~J}^{4}=1.8 \mathrm{~Hz}\right) ;{ }^{13} \mathbf{C} \mathbf{N M R}\left(\mathbf{1 0 0} \mathbf{M H z}, \mathbf{C D C l}_{\mathbf{3}}, \mathbf{2 5}^{\circ} \mathbf{C}\right) \delta: 14.3,61.1$,

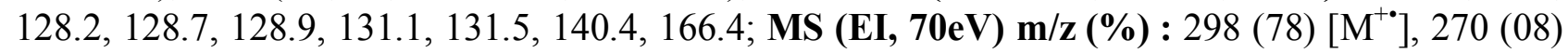


$\left[\mathrm{M}^{+\bullet}-\mathrm{CO}\right], 253(100) \quad\left[\mathrm{M}^{+\bullet}-\mathrm{OC}_{2} \mathrm{H}_{5}\right], 242(09), 225$ (20) $\left[\mathrm{M}^{+\bullet}-\mathrm{COOC}_{2} \mathrm{H}_{5}\right], 152$ (46) $\left[\mathrm{M}^{+\bullet}-\right.$ $2 \mathrm{COOC}_{2} \mathrm{H}_{5}$ ], 104 (07), 76 (07) ; HRMS : calculated for $\mathrm{C}_{18} \mathrm{H}_{18} \mathrm{O}_{4}$ : 298.1205, found 298.1207; Elemental Analysis calculated : C $72.47 \%$, H $6.08 \%$, O $21.45 \%$; found C $72.46 \%$, H $6.07 \%$, O $21.47 \%$.

2,2'-dinitrobiphenyl ${ }^{\mathbf{1 2}}$ (2p): beige solid; m.p. $120-122^{\circ} \mathrm{C}$ (Lit. $\left.123-128^{\circ} \mathrm{C}\right)$; IR (Nujol) $\boldsymbol{v}=1600$, 1567, 1520, 1353, 1299, 1254, 850, 786, 756, 746, 680, $588 \mathrm{~cm}^{-1} ;{ }^{1} \mathbf{H}_{\text {NMR (400 MHz, CDCl}}$, $\left.\mathbf{2 5}^{\circ} \mathbf{C}\right) \delta: 7.29\left(1 \mathrm{H}, \mathrm{dd}, \mathrm{J}^{3}=7.4 \mathrm{~Hz}, \mathrm{~J}^{4}=1.4 \mathrm{~Hz}\right), 7.58\left(1 \mathrm{H}, \mathrm{ddd}, \mathrm{J}^{3}=8.2 \mathrm{~Hz}, \mathrm{~J}^{3}=7.4 \mathrm{~Hz}, \mathrm{~J}^{4}=1.4 \mathrm{~Hz}\right), 7.68$ $\left(1 \mathrm{H}, \mathrm{ddd}, \mathrm{J}^{3}=7.4 \mathrm{~Hz}, \mathrm{~J}^{3}=7.4 \mathrm{~Hz}, \mathrm{~J}^{4}=1.4 \mathrm{~Hz}\right), 8.20\left(1 \mathrm{H}, \mathrm{dd}, \mathrm{J}^{3}=8.2 \mathrm{~Hz}, \mathrm{~J}^{4}=1.4 \mathrm{~Hz}\right){ }^{13} \mathbf{C}$ NMR (100 MHz, $\left.\mathbf{C D C l}_{3}, \mathbf{2 5}^{\circ} \mathbf{C}\right) \delta: 124.7,129.1,130.9,133.4,134.1,147.0 ;$ MS (EI, 70eV) $\mathbf{~ m} / \mathbf{z}(\%): 198(100)$ $\left[\mathrm{M}^{+\bullet}-\mathrm{NO}_{2}\right], 180(28), 168(41), 152$ (33), 139 (47), 126 (09), 115 (28), 102 (05), 89 (08), 76 (10), 63 (13), $51(06)$.

2-fluoro-3-carbethoxy-4-iodopyridine (3q): coulorless oil; IR (Neat) $v=2995,1739,1592,1551$, 1448, 1412, 1372, 1293, 1236, 1182, 1124, 1068, 1021, 890, $702 \mathrm{~cm}^{-1} ;{ }^{\mathbf{1}} \mathbf{H}$ NMR (400 MHz, $\left.\mathbf{C D C l}_{3}, \mathbf{2 5}^{\circ} \mathbf{C}\right) \delta: 1.43\left(3 \mathrm{H}, \mathrm{t}, \mathrm{J}^{3}=7.3 \mathrm{~Hz}\right), 4.47\left(2 \mathrm{H}, \mathrm{q}, \mathrm{J}^{3}=7.3 \mathrm{~Hz}\right), 7.71\left(1 \mathrm{H}, \mathrm{d}, \mathrm{J}^{3}=5.0 \mathrm{~Hz}\right), 7.92(1 \mathrm{H}$, $\left.\mathrm{d}, \mathrm{J}^{3}=5.0 \mathrm{~Hz}\right) ;{ }^{13} \mathbf{C}$ NMR (100 $\left.\mathbf{M H z} \mathbf{C D C l}_{3}, \mathbf{2 5}^{\circ} \mathbf{C}\right) \delta: 13.9,62.6,107.1\left(\mathrm{~d}, \mathrm{~J}^{3}(\mathrm{C}-\mathrm{F})=2.9 \mathrm{~Hz}\right), 123.0$ $\left(\mathrm{d}, \mathrm{J}^{2}(\mathrm{C}-\mathrm{F})=34.5 \mathrm{~Hz}\right), 132.0 \quad\left(\mathrm{~d}, \mathrm{~J}^{4}(\mathrm{C}-\mathrm{F})=4.8 \mathrm{~Hz}\right), 148.2 \quad\left(\mathrm{~d}, \mathrm{~J}^{3}(\mathrm{C}-\mathrm{F})=16.3 \mathrm{~Hz}\right), 158.6 \quad\left(\mathrm{~d}, \mathrm{~J}^{1}(\mathrm{C}-\right.$ $\mathrm{F})=244 \mathrm{~Hz}), 163.4\left(\mathrm{~d}, \mathrm{~J}^{3}(\mathrm{C}-\mathrm{F})=4.8 \mathrm{~Hz}\right) ; \mathbf{R M N}^{19} \mathbf{F}\left(376 \mathbf{M H z} \mathbf{C D C l}_{3}, \mathbf{2 5}^{\circ} \mathbf{C}\right) \delta:-65.4(\mathrm{~s})$; $\mathbf{M S}(\mathbf{E I}$, $\mathbf{7 0 e V}) \mathbf{~ m} / \mathbf{z}(\%): 295$ (47) $\left[\mathrm{M}^{+\bullet}\right], 267(54)\left[\mathrm{M}^{+\bullet}-\mathrm{C}_{2} \mathrm{H}_{4}\right], 250(100)\left[\mathrm{M}^{+\bullet}-\mathrm{OC}_{2} \mathrm{H}_{5}\right], 222$ (20) $\left[\mathrm{M}^{+\bullet}-\right.$ $\mathrm{COOC}_{2} \mathrm{H}_{5}$ ], 195 (09), 123 (07), 95 (07), 68 (11); HRMS : calculated for $\mathrm{C}_{8} \mathrm{H}_{7} \mathrm{FINO}_{2}$ : 294.9506, found 294.9502. Elemental Analysis calculated : $32.57 \%$, H $2.39 \%$, N $4.75 \%$; found C $32.34 \%$, H $2.31 \%$, N $4.64 \%$.

2,2'-difluoro-3,3'-dicarbethoxy-4,4'-bipyridyl (2q): yellow solid; m.p. $84-86^{\circ} \mathrm{C}$; IR (KBr) $\boldsymbol{v}=$ 2983, 1725, 1598, 1542, 1476, 1411, 1382, 1327, 1293, 1242, 1180, 1152, 1081, 1021, 852, 796, 664, $589 \mathrm{~cm}^{-1} ;{ }^{1} \mathbf{H}$ NMR (400 $\left.\mathbf{~ M H z}, \mathbf{C D C l}_{3}, \mathbf{2 5}^{\circ} \mathbf{C}\right) \delta: 1.12\left(3 \mathrm{H}, \mathrm{t}, \mathrm{J}^{3}=7.1 \mathrm{~Hz}\right), 4.18(2 \mathrm{H}, \mathrm{q}$, $\left.\mathrm{J}^{3}=7.1 \mathrm{~Hz}\right), 7.16\left(1 \mathrm{H}, \mathrm{d}, \mathrm{J}^{3}=5.0 \mathrm{~Hz}\right), 8.38\left(1 \mathrm{H}, \mathrm{d}, \mathrm{J}^{3}(\mathrm{H})=5.0 \mathrm{~Hz}\right) ;{ }^{13} \mathbf{C}$ NMR $\left(\mathbf{1 0 0} \mathbf{M H z}, \mathbf{C D C l}_{3}, \mathbf{2 5}^{\circ} \mathbf{C}\right)$ $\delta: 13.4,62.0,114.4\left(\mathrm{~d}, \mathrm{~J}^{2}(\mathrm{C}-\mathrm{F})=30.7 \mathrm{~Hz}\right), 121.7\left(\mathrm{~d}, \mathrm{~J}^{4}(\mathrm{C}-\mathrm{F})=4.8 \mathrm{~Hz}\right), 149.1\left(\mathrm{~d}, \mathrm{~J}^{3}(\mathrm{C}-\mathrm{F})=16.3 \mathrm{~Hz}\right)$, $150.1,160.4\left(\mathrm{~d}, \mathrm{~J}^{1}(\mathrm{C}-\mathrm{F})=160.5 \mathrm{~Hz}\right), 162.3\left(\mathrm{~d}, \mathrm{~J}^{3}(\mathrm{C}-\mathrm{F})=5.8 \mathrm{~Hz}\right) ;{ }^{19} \mathbf{F}$ NMR (376 $\left.\mathbf{~ M H z}, \mathbf{C D C l}_{3}, \mathbf{2 5}^{\circ} \mathbf{C}\right)$

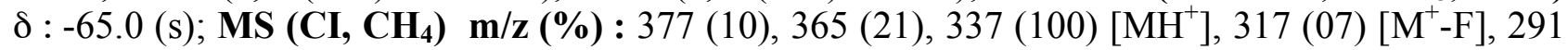
(78) $\left[\mathrm{M}^{+}-\mathrm{OC}_{2} \mathrm{H}_{5}\right], 263$ (11) $\left[\mathrm{M}^{+}-\mathrm{COOC}_{2} \mathrm{H}_{5}\right], 245$ (05), 219 (05); HRMS : calculated for $\mathrm{C}_{16} \mathrm{H}_{15} \mathrm{~F}_{2} \mathrm{~N}_{2} \mathrm{O}_{4}: 337.1000$, found 337.0997 ; Elemental Analysis calculated : $57.14 \%, \mathrm{H} \mathrm{4.20 \% ,} \mathrm{N}$ $8.33 \%$, F $11.30 \%$; found C $57.30 \%$, H $4.22 \%$, N $8.26 \%$, F $11.26 \%$.

1,2-bis(2-iodophenyl)ethane ${ }^{\mathbf{1 3}}$ (4): white solid; m.p. $101-103^{\circ} \mathrm{C}$; IR (KBr) $v=2946,2932,2861$, 1462, 1428, 1293, 1204, 1152, 1091, 1048, 1012, 951, 861, 753, 716, 646, $538 \mathrm{~cm}^{-1}$; ${ }^{\mathbf{H}}$ NMR (400 MHz, $\left.\mathbf{C D C l}_{3}, \mathbf{2 5}^{\circ} \mathbf{C}\right) \delta: 2.99(2 \mathrm{H}, \mathrm{s}), 6.90\left(1 \mathrm{H}, \mathrm{ddd}, \mathrm{J}^{3}=7.8 \mathrm{~Hz}, \mathrm{~J}^{3}=7.8 \mathrm{~Hz}, \mathrm{~J}^{4}=1.8 \mathrm{~Hz}\right), 7.19-7.31(2 \mathrm{H}$, $\mathrm{m}), 7.83\left(1 \mathrm{H}, \mathrm{d}, \mathrm{J}^{3}=7.8 \mathrm{~Hz}\right) ;{ }^{13} \mathbf{C}$ NMR (100 $\left.\mathbf{~ M H z}, \mathbf{C D C l}_{\mathbf{3}}, \mathbf{2 5}^{\circ} \mathbf{C}\right) \delta: 41.2,100.6,128.0,128.3$,

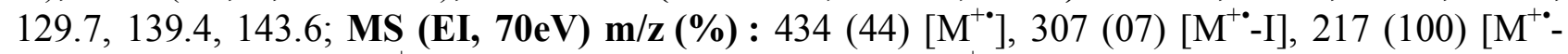
$\left.\mathrm{C}_{6} \mathrm{H}_{4} \mathrm{ICH}_{2}\right], 180(67)\left[\mathrm{M}^{+\bullet}-2 \mathrm{I}\right], 165$ (15), $152(06), 90(23)\left[\mathrm{M}^{+\bullet}-\mathrm{C}_{6} \mathrm{H}_{4} \mathrm{ICH}_{2}-\mathrm{I}\right], 63(05)$.

9,10-dihydrophenanthrene $\left.\mathbf{1 4}^{\mathbf{1 4}} \mathbf{6}\right)$ : colourless liquid; IR (Neat) $v=3068,3030,2941,2898,2838$, 2359, 1489, 1458, 1449, 779, 749, 730, $622 \mathrm{~cm}^{-1}$; ${ }^{\mathbf{H}} \mathbf{H} \mathbf{N M R}\left(\mathbf{4 0 0} \mathbf{~ M H z}, \mathbf{C D C l}_{\mathbf{3}}, \mathbf{2 5}^{\circ} \mathbf{C}\right) \delta: 2.87(2 \mathrm{H}$, s), 7.20-7.26 (2H, m), 7.26-7.33 (1H, m), $7.74\left(1 \mathrm{H}, \mathrm{d}, \mathrm{J}^{3}=7.8 \mathrm{~Hz}\right) ;{ }^{13} \mathbf{C}$ NMR (100 MHz, CDCl $\mathbf{~ M}_{3}$, $\left.\mathbf{2 5}^{\circ} \mathbf{C}\right) \delta: 29.0,123.7,126.9,127.3,128.1,134.5,137.4$; $\mathbf{M S}(\mathbf{E I}, \mathbf{7 0 e V}) \mathbf{~ m} / \mathbf{z}(\%): 180(100)\left[\mathrm{M}^{+*}\right]$, $165(33)\left[\mathrm{M}^{+\bullet}-\mathrm{CH}_{3}\right], 152(12)\left[\mathrm{M}^{+\bullet}-\mathrm{C}_{2} \mathrm{H}_{4}\right], 89(10), 76(05)$.

6-bromopiperonal ${ }^{\mathbf{1 5}}$ (7): white solid, m.p. $124-126^{\circ} \mathrm{C}$ (Lit. $131^{\circ} \mathrm{C}$ ); IR $(\mathbf{K B r}) \boldsymbol{v}=2866,1678$, $1617,1598,1490,1415,1392,1345,1265,1218,1115,1030,979,930,890,842,782,679,602$, $517 \mathrm{~cm}^{-1} ;{ }^{1} \mathbf{H}$ NMR (400 MHz, $\left.\mathbf{C D C l}_{3}, \mathbf{2 5}^{\circ} \mathbf{C}\right) \delta: 6.09(2 \mathrm{H}, \mathrm{s}), 7.05(1 \mathrm{H}, \mathrm{s}), 7.34(1 \mathrm{H}, \mathrm{s}), 10.17$ $(1 \mathrm{H}, \mathrm{s}) ;{ }^{13} \mathbf{C} \mathbf{N M R}\left(\mathbf{1 0 0} \mathbf{M H z}, \mathbf{C D C l}_{3}, \mathbf{2 5}^{\circ} \mathbf{C}\right) \delta: 102.7,108.0,113.2,121.5,127.9,148.1,153.3$, 


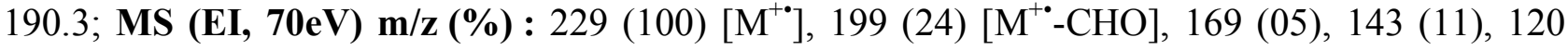
(10), 90 (05), 62 (23), 50 (05).

6-iodopiperonal ${ }^{\mathbf{1 6}}(\mathbf{8})$ : yellow solid, m.p. $102-103^{\circ} \mathrm{C}\left(\right.$ Lit. $\left.102-104^{\circ} \mathrm{C}\right)$; IR $(\mathbf{K B r}) \boldsymbol{v}=2894,1674$, 1612, 1594, 1508, 1486, 1406, 1386, 1345, 1256, 1115, 1045, 970, 932, 876, 847, 786, 663, 607 $\mathrm{cm}^{-1} ;{ }^{1} \mathbf{H}$ NMR (400 MHz, $\left.\mathbf{C D C l}_{3}, \mathbf{2 5}^{\circ} \mathbf{C}\right) \delta: 6.09(2 \mathrm{H}, \mathrm{s}), 7.32(1 \mathrm{H}, \mathrm{s}), 7.35(1 \mathrm{H}, \mathrm{s}), 9.87(1 \mathrm{H}, \mathrm{s})$; ${ }^{13} \mathbf{C}$ NMR (100 $\left.\mathbf{M H z} \mathbf{C D C l}_{3}, \mathbf{2 5}^{\circ} \mathbf{C}\right) \delta: 93.3,102.6,108.7,119.3,129.5,149.1,153.4,194.4$; MS

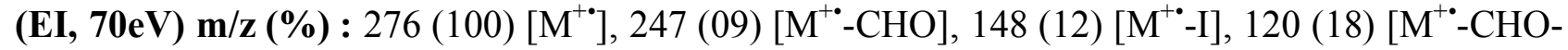
I], 62 (11).

2,2'-diiodo- $N$-methyl-4,5-methylenedioxybenzanilide (9): white solid, m.p. $213-214^{\circ} \mathrm{C}$; IR (KBr) $v=1645,1575,1505,1485,1425,1387,1359,1303,1237,1124,1101,1040,922,865,847$, $763,725,683,566 \mathrm{~cm}^{-1}$; Owning to the restricted rotation around the amide bond, two rotamers are observed (ratio $70: 30$ ) at $25^{\circ} \mathrm{C}$ in $\mathrm{CDCl}_{3}:{ }^{1} \mathbf{H} \mathbf{~ N M R}\left(400 \mathbf{~ M H z}, \mathbf{C D C l}_{3}, \mathbf{2 5}^{\circ} \mathbf{C}\right) \delta: 3.12(0.9 \mathrm{H}, \mathrm{s})$ and $3.38(2.1 \mathrm{H}, \mathrm{s}), 5.85,5.87\left(1.4 \mathrm{H}, 2 \mathrm{~d}, \mathrm{~J}^{2}=1.4 \mathrm{~Hz}\right)$ and $6.03,6.05\left(0.6 \mathrm{H}, 2 \mathrm{~d}, \mathrm{~J}^{2}=1.4 \mathrm{~Hz}\right), 6.86(0.7 \mathrm{H}$, s) and $7.01(0.3 \mathrm{H}, \mathrm{s}), 6.92\left(0.7 \mathrm{H}, \mathrm{ddd}, \mathrm{J}^{3}=7.8 \mathrm{~Hz}, \mathrm{~J}^{3}=7.3 \mathrm{~Hz}, \mathrm{~J}^{4}=1.8 \mathrm{~Hz}\right)$ and $7.06-7.13(0.3 \mathrm{H}, \mathrm{m}), 7.10$ $(0.7 \mathrm{H}, \mathrm{s})$ and $7.27(0.3 \mathrm{H}, \mathrm{s}), 7.22\left(0.7 \mathrm{H}, \mathrm{ddd}, \mathrm{J}^{3}=7.8 \mathrm{~Hz}, \mathrm{~J}^{3}=7.3 \mathrm{~Hz}, \mathrm{~J}^{4}=1.4 \mathrm{~Hz}\right)$ and $7.44-7.54(0.3 \mathrm{H}$, $\mathrm{m}), 7.48\left(1 \mathrm{H}, \mathrm{dd}, \mathrm{J}^{3}=8.2 \mathrm{~Hz}, \mathrm{~J}^{3}=7.8 \mathrm{~Hz}, \mathrm{~J}^{4}=1.8 \mathrm{~Hz}\right), 7.82\left(0.7 \mathrm{H}, \mathrm{dd}, \mathrm{J}^{3}=7.8 \mathrm{~Hz}, \mathrm{~J}^{4}=1.4 \mathrm{~Hz},\right)$ and 7.93 $\left(0.3 \mathrm{H}, \mathrm{d}, \mathrm{J}^{3}=7.3 \mathrm{~Hz}\right) ;{ }^{13} \mathbf{C}$ NMR $\left(\mathbf{1 0 0} \mathbf{M H z}, \mathbf{C D C l}_{\mathbf{3}}, \mathbf{2 5}^{\circ} \mathbf{C}\right) \delta: 36.9$ and $39.4,81.0$ and $83.1,98.1$ and 98.7, 101.7 and 102.0, 107.2 and 107.7, 118.4, 118.7, 128.6, 129.48, 129.50, 129.59, 129.9, 135.2 and 135.4, 139.7 and 140.0, 144.8, 145.6, 147.5, 148.47, 148.53, 148.9, 169.4 and 169.5; MS (EI, 70eV) m/z (\%) : $507(06)\left[\mathrm{M}^{+\bullet}\right], 380(100)\left[\mathrm{M}^{+\bullet}-\mathrm{I}\right], 275(90)\left[\mathrm{M}^{+\bullet}-\mathrm{IC}_{6} \mathrm{H}_{4} \mathrm{NCH}_{3}\right], 253(15), 247$ (11) $\left[\mathrm{M}^{+\bullet}-\mathrm{IC}_{6} \mathrm{H}_{4} \mathrm{NCH}_{3}-\mathrm{CO}\right.$ ], 120 (12), 62 (05); HRMS : calculated for $\mathrm{C}_{15} \mathrm{H}_{12} \mathrm{O}_{3} \mathrm{NI}_{2}$ : 507.8907, found 507.8904. Elemental Analysis calculated : C $35.53 \%, \mathrm{H} 2.19 \%$, N $2.76 \%$; found C 35.32 $\%$, H $2.18 \%$, N $2.67 \%$.

N-methylcrinasiadine ${ }^{17}$ (11): pale yellow solid, m.p. $241-243^{\circ} \mathrm{C}$ (Lit. $247.5-248.5^{\circ} \mathrm{C}$ ); IR (KBr) v $=2922,1658,1622,1608,1499,1463,1415,1400,1350,1317,1264,1250,1209,1099,1039,937$, 880, 847, 753, 683, 627, $594 \mathrm{~cm}^{-1} ;{ }^{1} \mathbf{H}$ NMR (400 $\left.\mathbf{~ M H z}, \mathbf{C D C l}_{3}, \mathbf{2 5}^{\circ} \mathbf{C}\right) \delta: 3.81(3 \mathrm{H}, \mathrm{s}), 6.13(2 \mathrm{H}$, s), $7.30\left(1 \mathrm{H}, \mathrm{ddd}, \mathrm{J}^{3}=7.6 \mathrm{~Hz}, \mathrm{~J}^{3}=7.6 \mathrm{~Hz}, \mathrm{~J}^{4}=0.9 \mathrm{~Hz}\right), 7.40\left(1 \mathrm{H}, \mathrm{d}, \mathrm{J}^{3}=8.3 \mathrm{~Hz}\right), 7.52\left(1 \mathrm{H}, \mathrm{ddd}, \mathrm{J}^{3}=8.7 \mathrm{~Hz}\right.$, $\left.\mathrm{J}^{3}=6.9 \mathrm{~Hz}, \mathrm{~J}^{4}=1.4 \mathrm{~Hz}\right), 7.62(1 \mathrm{H}, \mathrm{s}), 7.91(1 \mathrm{H}, \mathrm{s}), 8.09\left(1 \mathrm{H}, \mathrm{dd}, \mathrm{J}^{3}=7.8 \mathrm{~Hz}, \mathrm{~J}^{4}=1.2 \mathrm{~Hz}\right) ;{ }^{13} \mathbf{C} \mathbf{N M R}(\mathbf{1 0 0}$ $\left.\mathbf{M H z}, \mathbf{C D C l}_{3}, \mathbf{2 5}^{\circ} \mathbf{C}\right) \delta: 30.0,100.4,101.9,107.0,115.0,119.2,121.3,122.3,122.9,128.9,130.4$,

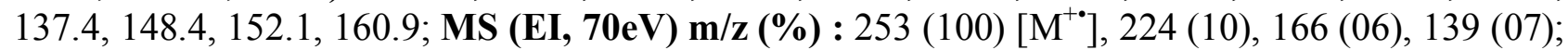
HRMS : calculated for $\mathrm{C}_{15} \mathrm{H}_{11} \mathrm{O}_{3} \mathrm{~N}: 253.0739$, found 253.0744 .

2-fluoro-3-iodopyridine $\mathbf{~}^{\mathbf{1 8}}$ (12): white solid ; m.p. $42-43^{\circ} \mathrm{C}$ (Lit. $\left.42^{\circ} \mathrm{C}\right)$; IR (KBr) $v=1582,1551$, 1425, 1401, 1251, 1063, 1021, 847, 804, 739, 650, $569 \mathrm{~cm}^{-1} ;{ }^{\mathbf{1}} \mathbf{H}$ NMR (400 $\left.\mathbf{M H z}, \mathbf{C D C l}_{3}, \mathbf{2 5}^{\circ} \mathbf{C}\right)$ $\delta$ : 6.94-7.01 $(1 \mathrm{H}, \mathrm{m}), 8.14-8.21(2 \mathrm{H}, \mathrm{m}) ;{ }^{13} \mathbf{C}$ NMR (100 $\left.\mathbf{~ M H z}, \mathbf{C D C l}_{3}, \mathbf{2 5}^{\circ} \mathbf{C}\right) \delta: 76.0\left(\mathrm{~d}, \mathrm{~J}^{2}(\mathrm{C}-\right.$ $\mathrm{F})=43 \mathrm{~Hz}), 122.7\left(\mathrm{~d}, \mathrm{~J}^{4}(\mathrm{C}-\mathrm{F})=3.8 \mathrm{~Hz}\right), 147.2\left(\mathrm{~d}, \mathrm{~J}^{3}(\mathrm{C}-\mathrm{F})=13.4 \mathrm{~Hz}\right), 150.1\left(\mathrm{~d}, \mathrm{~J}^{3}(\mathrm{C}-\mathrm{F})=2.9 \mathrm{~Hz}\right), 162.0$ $\left(\mathrm{d}, \mathrm{J}^{1}(\mathrm{C}-\mathrm{F})=235 \mathrm{~Hz}\right)$; $\mathbf{R M N}{ }^{19} \mathbf{F}\left(376 \mathbf{M H z} \mathbf{C D C l}_{3}, \mathbf{2 5}^{\circ} \mathrm{C}\right) \delta:-54.4(\mathrm{~s}) ; \mathbf{M S}(\mathbf{E I}, \mathbf{7 0 e V}) \mathbf{~ m} / \mathbf{z}(\%)$ : $223(100)\left[\mathrm{M}^{+\bullet}\right], 127(06)\left[\mathrm{M}^{+\bullet}-\mathrm{C}_{5} \mathrm{H}_{3} \mathrm{NF}\right], 96(22)\left[\mathrm{M}^{+\bullet}-\mathrm{I}\right], 76(10)\left[\mathrm{M}^{+\bullet}-\mathrm{I}-\mathrm{HF}\right], 73(10)$.

2-bromo-1-(bromomethyl)benzene ${ }^{\mathbf{1 9}}$ (13): colourless liquid ; b.p. $74^{\circ} \mathrm{C}$ under 0.30 Torr (Lit. 120 $130^{\circ} \mathrm{C}$ under 13 Torr); IR (Neat) $v=661,728,758,1030,1051,1224,1445,1482,1568,1690$, $3063 \mathrm{~cm}^{-1} ;{ }^{1} \mathbf{H}$ NMR (400 MHz, $\left.\mathbf{C D C l}_{3}, \mathbf{2 5}^{\circ} \mathbf{C}\right) \delta: 4.60(2 \mathrm{H}, \mathrm{s}), 7.16\left(1 \mathrm{H}, \mathrm{ddd}, \mathrm{J}^{3}=7.8 \mathrm{~Hz}, \mathrm{~J}^{3}=7.8 \mathrm{~Hz}\right.$, $\left.\mathrm{J}^{4}=1.4 \mathrm{~Hz}\right), 7.29\left(1 \mathrm{H}, \mathrm{dd}, \mathrm{J}^{3}=7.8 \mathrm{~Hz}, \mathrm{~J}^{3}=7.8 \mathrm{~Hz}\right), 7.44\left(1 \mathrm{H}, \mathrm{dd}, \mathrm{J}^{3}=7.8 \mathrm{~Hz}, \mathrm{~J}^{4}=1.4 \mathrm{~Hz}\right), 7.56(1 \mathrm{H}, \mathrm{d}$, $\left.\mathrm{J}^{3}=7.8 \mathrm{~Hz}\right) ;{ }^{13} \mathbf{C}$ NMR $\left(\mathbf{1 0 0} \mathbf{M H z}_{\mathbf{C}} \mathbf{C D C l}_{3}, \mathbf{2 5}^{\circ} \mathbf{C}\right) \delta: 33.4,124.4,127.9,130.1,131.2,133.3,136.9$; MS (EI, 70eV) m/z (\%) : $250(20)\left[\mathrm{M}^{+\bullet}\right], 171 \& 169$ (96 \& 100) [ $\left.\mathrm{M}^{+\bullet}-\mathrm{Br}\right], 90(35)\left[\mathrm{M}^{+\bullet}-2 \mathrm{Br}\right], 63$ (14).

1,2-bis(2-bromophenyl)ethane ${ }^{20}(\mathbf{1 4})$ : white solid; m.p. $84-85^{\circ} \mathrm{C}$ (Lit. $\left.84-86^{\circ} \mathrm{C}\right)$; IR (KBr) $\boldsymbol{v}=$ 2932, 1566, 1472, 1453, 1435, 1293, 1156, 1096, 1040, 1021, 951, 870, 809, 753, 721, 655, 546 $\mathrm{cm}^{-1}{ }^{1}{ }^{1} \mathrm{H}$ NMR (400 MHz, $\left.\mathbf{C D C l}_{3}, \mathbf{2 5}^{\circ} \mathbf{C}\right) \delta: 3.03(2 \mathrm{H}, \mathrm{s}), 7.01-7.09(1 \mathrm{H}, \mathrm{m}), 7.14-7.23(2 \mathrm{H}, \mathrm{m})$, 
$7.53\left(1 \mathrm{H}, \mathrm{d}, \mathrm{J}^{3}=8.2 \mathrm{~Hz}\right) ;{ }^{13} \mathbf{C}$ NMR (100 $\left.\mathbf{M H z}, \mathbf{C D C l}_{3}, \mathbf{2 5}^{\circ} \mathbf{C}\right) \delta: 36.4,124.5,127.4,127.8,130.6$,

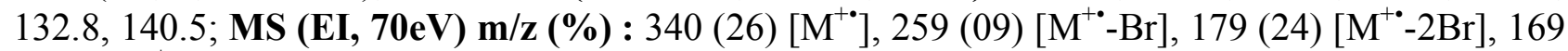
(100) $\left[\mathrm{M}^{+*}-\mathrm{BrC}_{6} \mathrm{H}_{4} \mathrm{CH}_{3}\right], 90(23), 63(06)$.

6-iodo-3,4-methylenedioxybenzoïc acid $^{\mathbf{2 1}}$ (15): white solid, m.p. $209-211^{\circ} \mathrm{C}$ (Lit. $216-217^{\circ} \mathrm{C}$ ); IR (KBr) $v=3758,2913,1702,1621,1505,1448,1420,1376,1359,1284,1256,1152,1120,1035$, 922, 885, 857, 786, 697, $617 \mathrm{~cm}^{-1} ;{ }^{1} \mathbf{H}$ NMR (400 MHz, DMSO-D6, 25 $\left.{ }^{\circ} \mathbf{C}\right) \delta: 6.09(2 \mathrm{H}, \mathrm{s}), 7.28$ $(1 \mathrm{H}, \mathrm{s}), 7.47(1 \mathrm{H}, \mathrm{s}) ;{ }^{13} \mathbf{C}$ NMR (100 MHz, DMSO-D6, 25$\left.{ }^{\circ} \mathbf{C}\right) \delta: 85.5,103.1,110.7,120.3,129.8$,

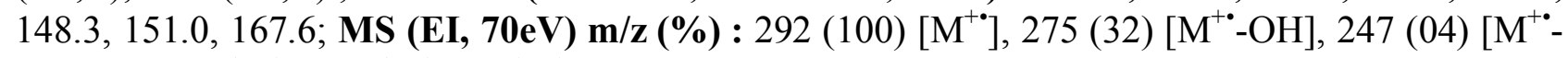
$\mathrm{COOH}], 165(05), 107$ (05), 62 (08).

2,2'-diiodo-4,5-methylenedioxybenzanilide (16): white solid, m.p. $182-183^{\circ} \mathrm{C}$; IR (KBr) $v=$ $3152,1645,1583,1533,1495,1476,1434,1401,1383,1345,1302,1242,1143,1035,932,744$, 702, 678, $556 \mathrm{~cm}^{-1} ;{ }^{1} \mathbf{H}$ NMR (400 MHz, $\left.\mathbf{C D C l}_{3}, \mathbf{2 5}^{\circ} \mathbf{C}\right) \delta: 6.06(2 \mathrm{H}, \mathrm{s}), 6.90\left(1 \mathrm{H}, \mathrm{ddd}, \mathrm{J}^{3}=7.3 \mathrm{~Hz}\right.$, $\left.\mathrm{J}^{3}=7.3 \mathrm{~Hz}, \mathrm{~J}^{4}=1.4 \mathrm{~Hz}\right), 7.08(1 \mathrm{H}, \mathrm{s}), 7.33(1 \mathrm{H}, \mathrm{s}), 7.40\left(1 \mathrm{H}, \mathrm{dd}, \mathrm{J}^{3}=7.8 \mathrm{~Hz}, \mathrm{~J}^{3}=7.8 \mathrm{~Hz}\right), 7.73(1 \mathrm{H}, \mathrm{s})$, $7.82\left(1 \mathrm{H}, \mathrm{dd}, \mathrm{J}^{3}=8.2 \mathrm{~Hz}, \mathrm{~J}^{4}=1.4 \mathrm{~Hz}\right), 8.36\left(1 \mathrm{H}, \mathrm{d}, \mathrm{J}^{3}=7.3 \mathrm{~Hz}\right) ;{ }^{13} \mathbf{C}$ NMR (100 MHz, CDCl $\left.3 \mathbf{~ 2 5}^{\circ} \mathbf{C}\right) \delta$ : 81.7, 90.3, 102.3, 108.7, 119.7, 122.2, 126.5, 129.3, 134.9, 137.9, 138.9, 148.4, 150.0, 166.7; MS (EI, 70eV) m/z (\%) : $493(13)\left[\mathrm{M}^{+\bullet}\right], 366(59)\left[\mathrm{M}^{+\bullet}-\mathrm{I}\right], 275(100)\left[\mathrm{M}^{+\bullet}-\mathrm{IC}_{6} \mathrm{H}_{4} \mathrm{NH}\right], 247(12)\left[\mathrm{M}^{+\bullet}-\right.$ $\left.\mathrm{IC}_{6} \mathrm{H}_{4} \mathrm{NH}-\mathrm{CO}\right], 239(12)\left[\mathrm{M}^{+\bullet}-2 \mathrm{I}\right], 120(13), 91$ (05), 62 (05). Elemental Analysis calculated : C $34.11 \%$, H $1.84 \%$, N $2.84 \%$; found C $33.93 \%$, H $1.78 \%$, N $2.74 \%$.

\footnotetext{
${ }^{1}$ Watson, S. C. J. Organometal. Chem. 1967, 9, 165.

${ }^{2}$ Koza, D. J.; Carita, E. Synthesis 2002, 15, 2183.

${ }^{3}$ Machiko, O.; Norio, Y.. Hiroyuki, A. Chem. Pharm. Bull. 1997, 45, 1745.

${ }_{5}^{4}$ Periasamy, M.; Jayakumar, K. N.; Bharathi,P. J. Org. Chem. 2000, 65, 3548.

${ }^{5}$ Tiecco, M.; Testaferri, L.; Tingoli, M.; Chanelli, D.; Montanucci, M. Synthesis 1984, 736; Lunn, G. J. Org. Chem. 1992, 57, 6317 .

${ }^{6}$ Courtois, V. ; Barhdadi, R. ; Troupel, M. ; Périchon, J. Tetrahedron 1997, 53, 11569.

${ }^{7}$ Wen, L.-S.; Kovacic, P. Tetrahedron 1978, 34, 2723.

${ }^{8}$ Jutand, A.; Mosleh, A. J. Org. Chem. 1997, 62, 261.

${ }^{9}$ Inoue, A.; Kitagawa, K.; Shinokubo, H.; Oshima, K. Tetrahedron 2000, 56, 9601.

${ }^{10}$ Johnson, F.; Nasutavicus, W. A. J. Heterocyclic Chem. 1965, 2, 26.

${ }^{11}$ Lee, P. H.; Seomoon, D.; Lee, K. Org. Lett. 2005, 7, 343.

12 The product has been compared to an authentic sample of commercial product purchased from Acros (CAS number: 2436-96-6 ; Product 15539-0000 )

${ }^{13}$ Khan, F. A.; Czerwonka, R.; Reissig, H.-U. Eur. J. Org. Chem. 2000, 3607

${ }^{14}$ Field, L. D.; Sternhell, S.; Wilton, H. V. Tetrahedron 1997, 53, 4051.

${ }_{15}$ Conrad, P. C.; Kwiatkowski, P. L.; Fuchs, P. L. J. Org. Chem. 1987, 52, 586.

${ }^{16}$ Bogucki, D. E.; Charlton, J. L. J. Org. Chem. 1995, 60, 588.

${ }^{17}$ Banwell, M. G.; Cowden, C. J. Aust. J. Chem. 1994, 47, 2235.

${ }_{18}^{18}$ Rocca, P.; Marsais, F.; Godard, A.; Quéguiner, G. Tetrahedron 1993, 49, 49.

${ }^{19}$ Shimizu, T.; Enomoto, M.; Taka, H.; Kamigata, N. J. Org. Chem. 1999, 64, 8242

${ }^{20}$ Aitken, R. A.; Hodgson, P. K. G.; Morrison, J. J.; Oyewale, A. O. J. Chem. Soc. Perkin Trans. $12002,402$.

${ }^{21}$ Padwa, A.; Brodney, M. A.; Lynch, S. M. J. Org. Chem. 2001, 66, 1716.
} 


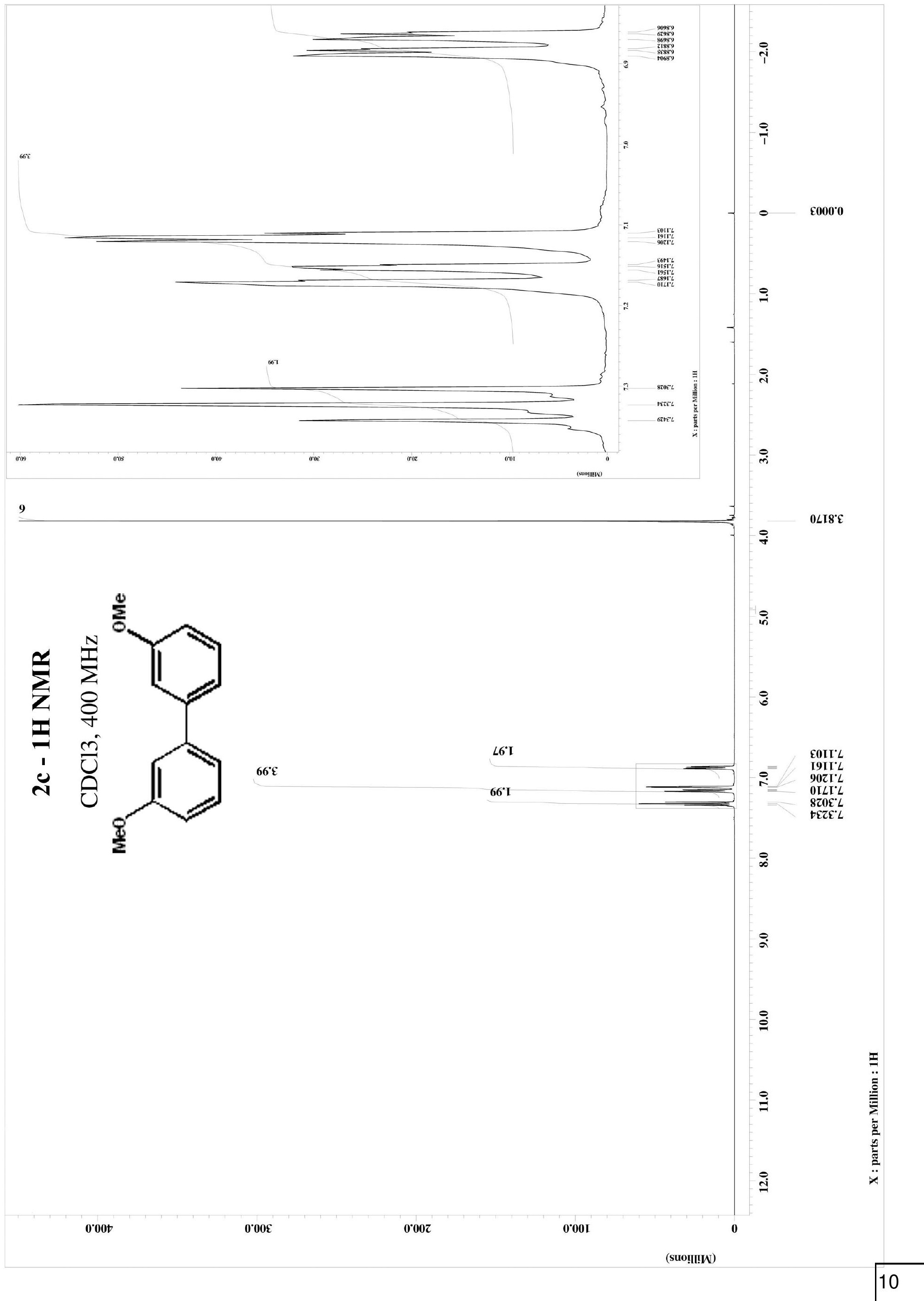




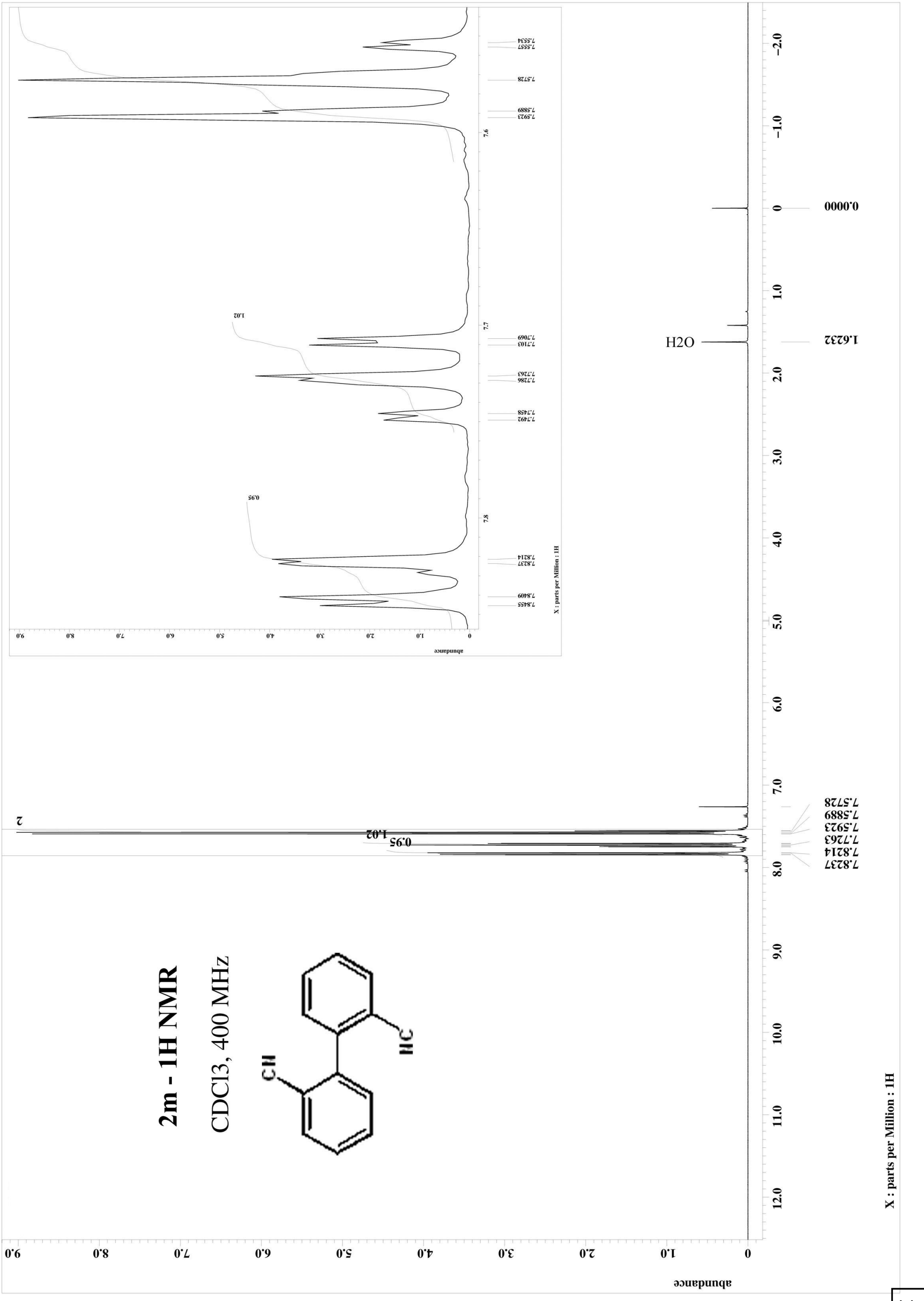




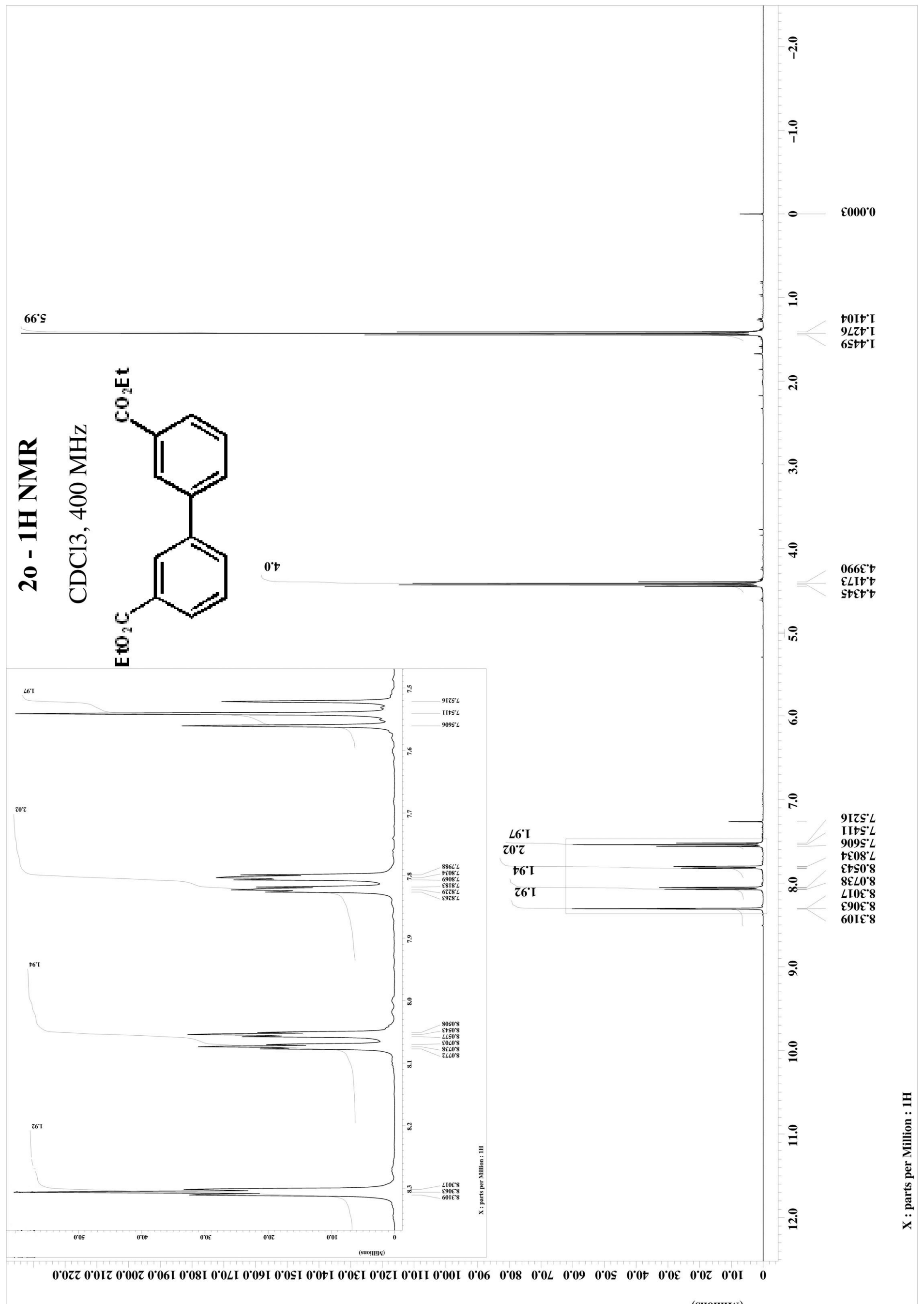




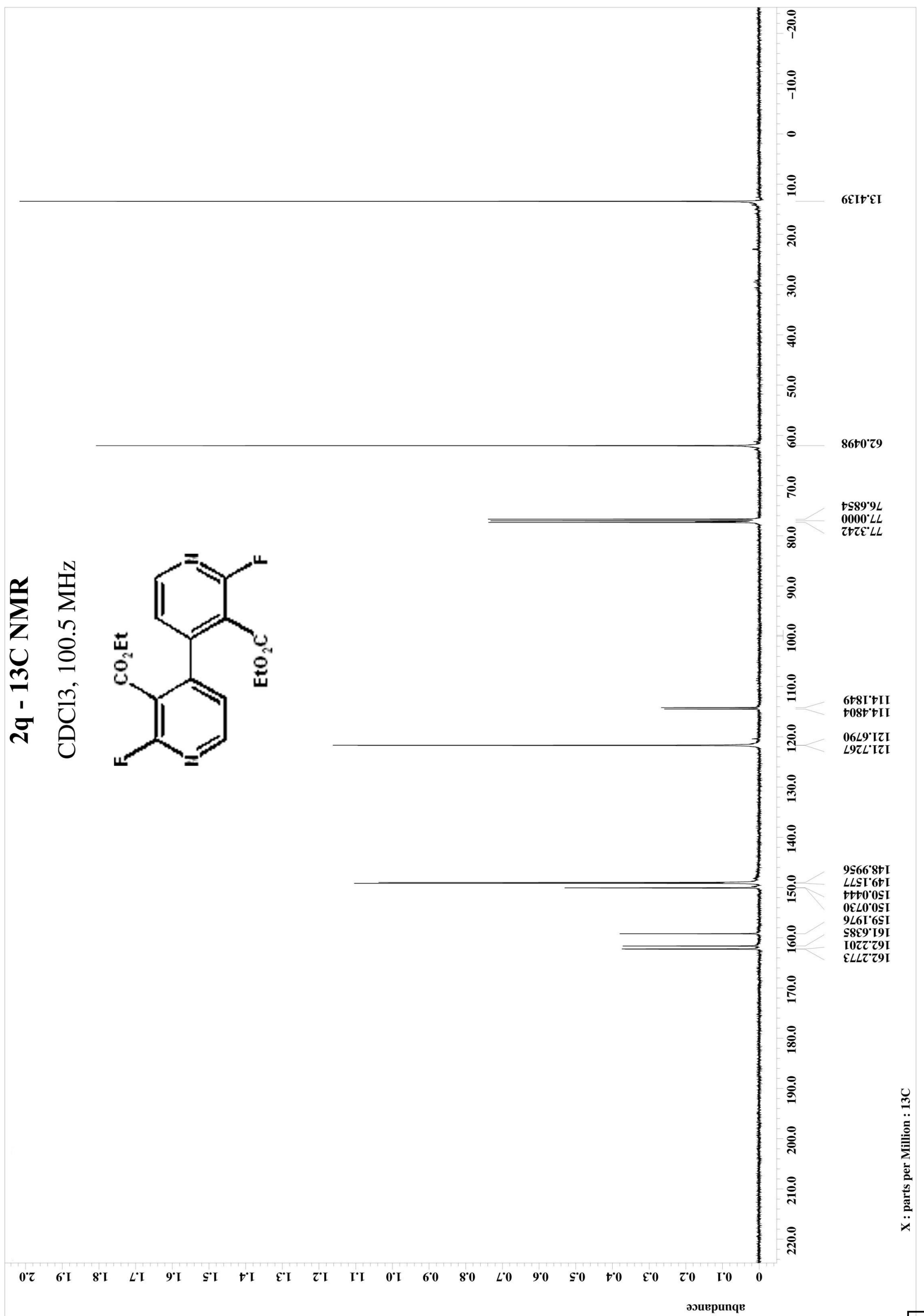




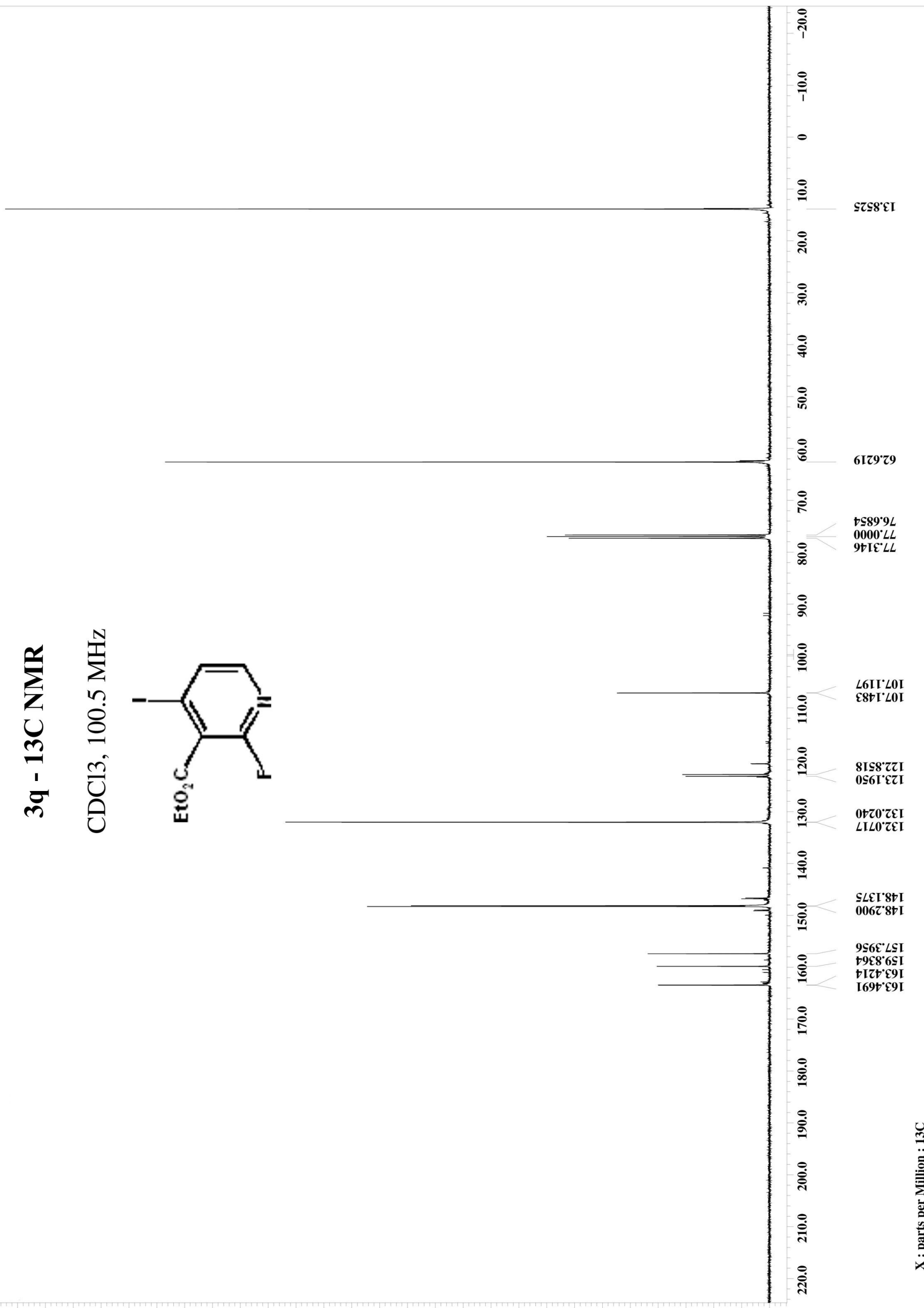

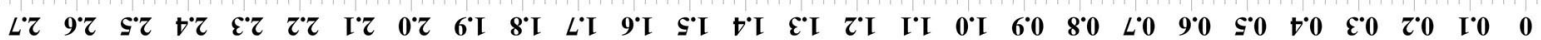




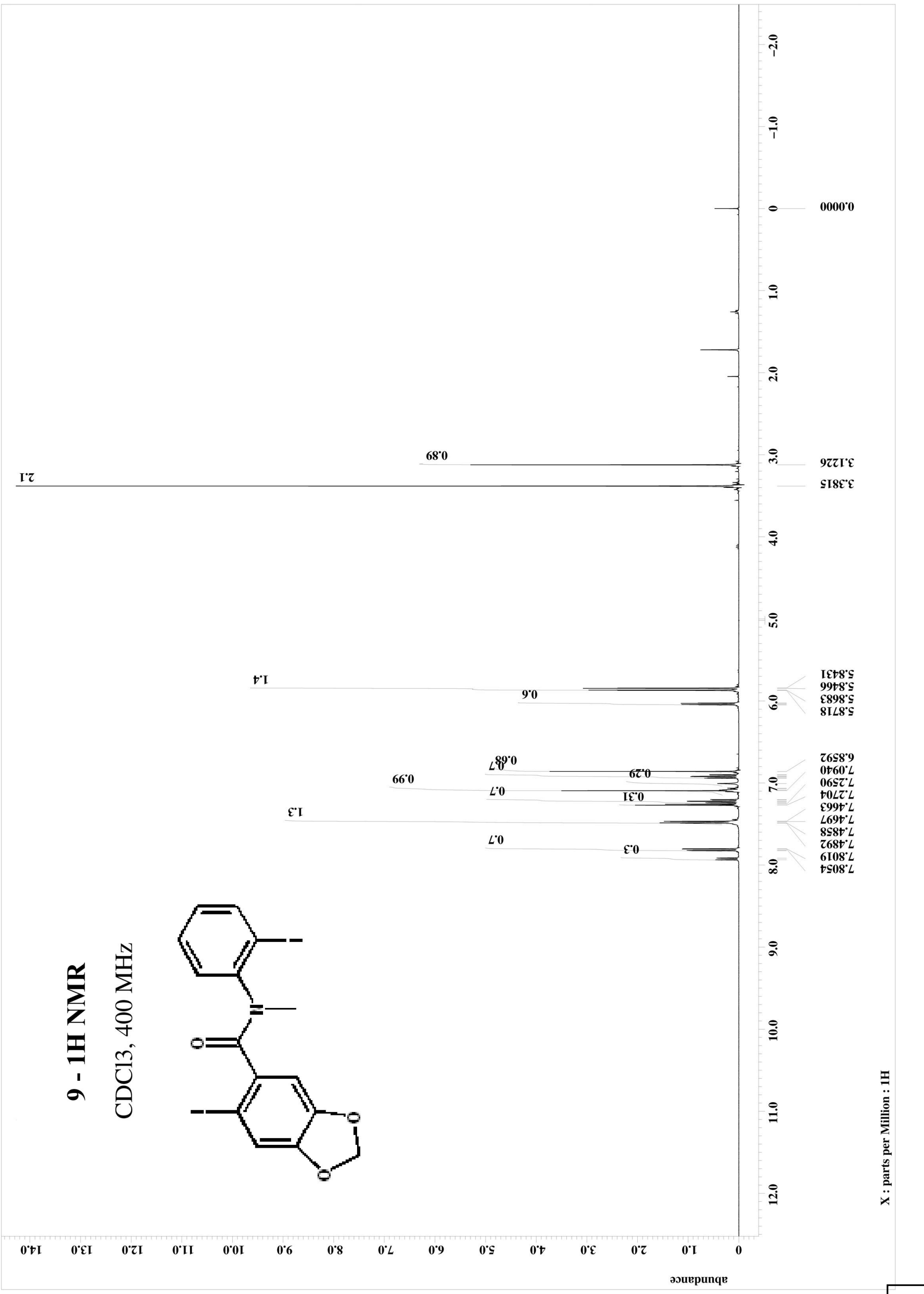



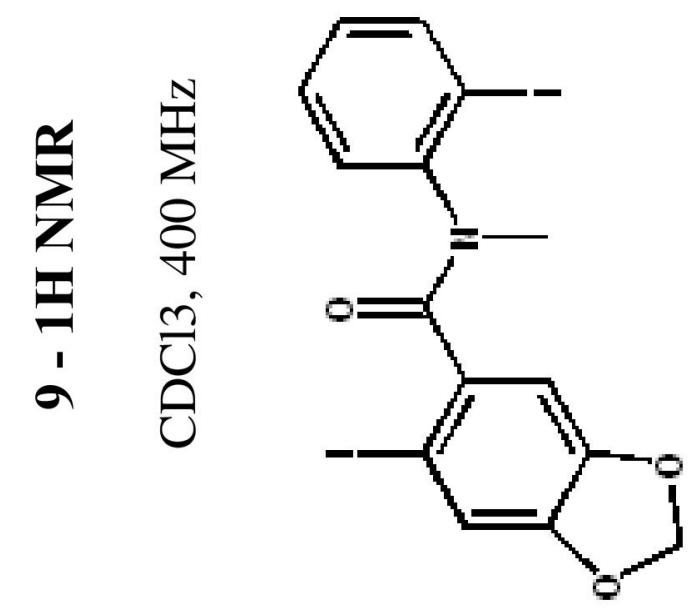

$9 \cdot 0$

$89^{\circ} 0$

L’0

660

$\varepsilon \cdot I$

L'0

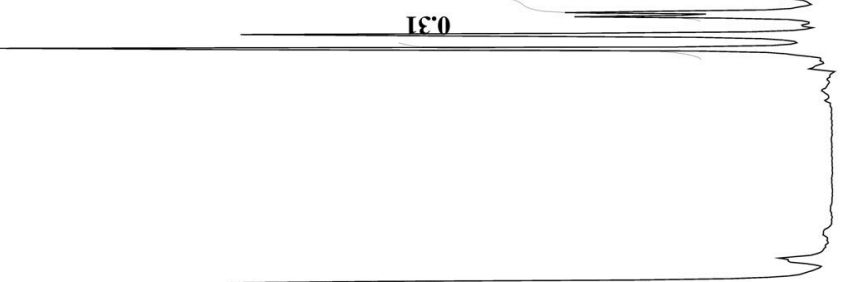

L'0

0

$\varepsilon * 0$ $\stackrel{\infty}{6}$

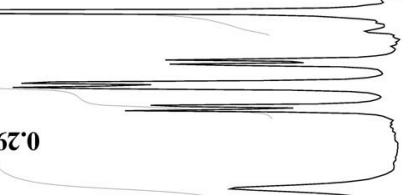

ㅂ. $\quad$ Esz09

$\angle 8200^{\circ} 9$
$8+t 00^{\circ}$

5

ชู

?.

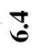

18

$\stackrel{\circ}{\circ}$

๑ิ

Z6ธ8.9

E668.9

$8816^{\circ} 9$

$z z z 6^{\circ} 9$

I $\angle E 6^{\circ} 9$

LIt6.9

$\stackrel{0}{\circ} \quad 0 \angle 00^{\circ} \mathrm{L}$

I $990^{\circ} \mathrm{L}$

$0_{0980}^{0} \angle$

$=\quad 0+60^{\circ} \mathrm{L}$

$\because \quad \begin{array}{ll}z I z Z^{\circ} L \\ ?\end{array}$

$900+2$

Ittr: $\tau^{\circ}$

$06 \subseteq 2<$

$\because \quad \quad \quad 0 \angle Z L$

$r$

$\stackrel{5}{r}$

E99t० $L$

$\angle 69)^{\circ} \angle$

$8 \leq 85^{\circ} \mathrm{L}$

$\stackrel{n}{n}$

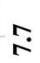

$\infty \quad 6 \mathrm{IOS}^{\circ} \mathrm{L}$

$+508^{\circ}$

tIZ8.

\begin{tabular}{l} 
S916 $L$ \\
0966 \\
\hline
\end{tabular} 


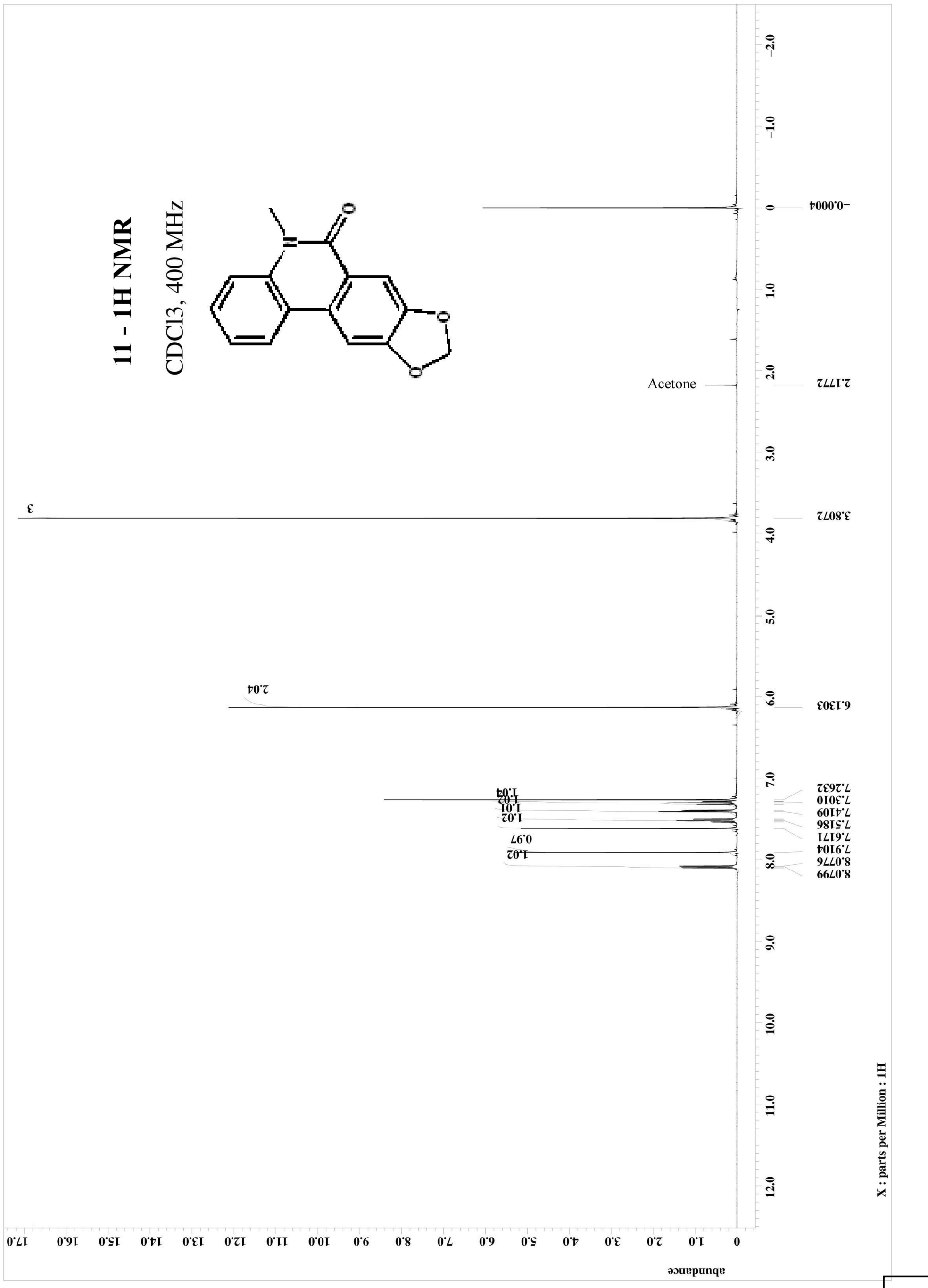




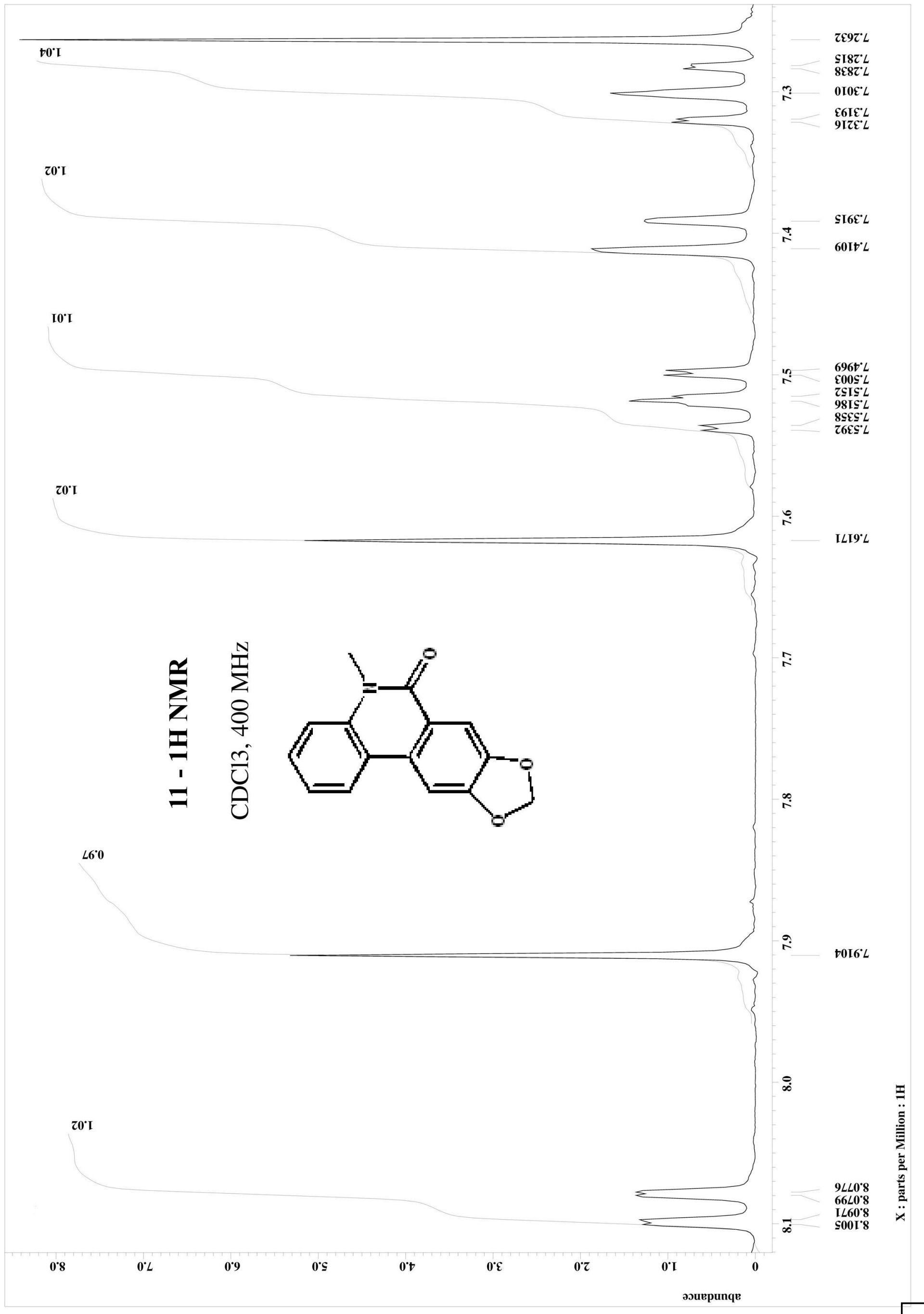




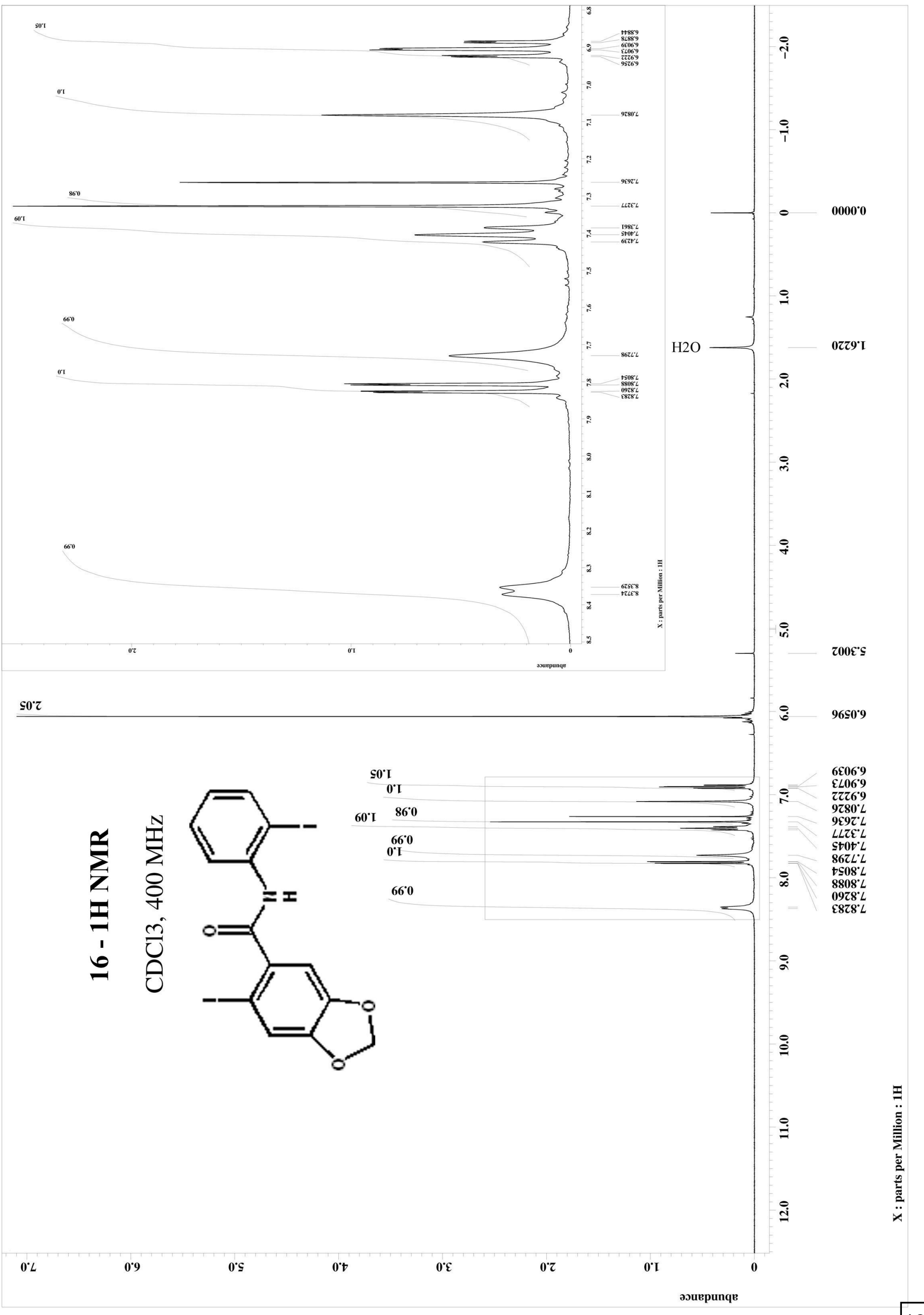

